\title{
PARETO LÉVY MEASURES AND MULTIVARIATE REGULAR VARIATION
}

\author{
IRMINGARD EDER * AND \\ CLAUDIA KLÜPPELBERG, ${ }^{* * *}$ Technische Universität München
}

\begin{abstract}
We consider regular variation of a Lévy process $\boldsymbol{X}:=\left(\boldsymbol{X}_{t}\right)_{t \geq 0}$ in $\mathbb{R}^{d}$ with Lévy measure $\Pi$, emphasizing the dependence between jumps of its components. By transforming the one-dimensional marginal Lévy measures to those of a standard 1-stable Lévy process, we decouple the marginal Lévy measures from the dependence structure. The dependence between the jumps is modeled by a so-called Pareto Lévy measure, which is a natural standardization in the context of regular variation. We characterize multivariate regularly variation of $\boldsymbol{X}$ by its one-dimensional marginal Lévy measures and the Pareto Lévy measure. Moreover, we define upper and lower tail dependence coefficients for the Lévy measure, which also apply to the multivariate distributions of the process. Finally, we present graphical tools to visualize the dependence structure in terms of the spectral density and the tail integral for homogeneous and nonhomogeneous Pareto Lévy measures.
\end{abstract}

Keywords: Dependence of Lévy processes; Lévy copula, Lévy measure; Pareto Lévy copula; multivariate regular variation; multivariate stable process; spectral measure; tail integral; tail dependence coefficient

2010 Mathematics Subject Classification: Primary 60E07; 60G51; 60G52

Secondary $60 \mathrm{G} 70$

\section{Introduction}

In a series of papers Hult and Lindskog [14]-[17] defined and investigated regular variation of measures and additive processes, which apply in particular to Lévy measures and Lévy processes. Their concept of regular variation of a stochastic process with càdlàg sample paths is, for a Lévy process $\boldsymbol{X}=\left(\boldsymbol{X}_{t}\right)_{t \geq 0}$, equivalent to regular variation of the random vector $\boldsymbol{X}_{1}$ and its Lévy measure; cf. [17, Theorem 5.1]. Similar concepts have been used to study the extremal behavior of stochastic processes in [7] and [13].

Since regular variation of a random vector $\boldsymbol{X}_{1}$ is well understood (cf. [23] and [24]), it seems that all relevant results can be translated from $\boldsymbol{X}_{1}$ to the corresponding Lévy measure. This is in principle true, and a distributional copula $C_{t}$ of $\boldsymbol{X}_{t}$ for every fixed $t$ could be invoked. However, owing to essential problems of this approach (for details, see [20, p. 1552]), alternatives have to be found. Tankov and collaborators (cf. [6, Section 5] and [20]) proposed dependence modeling of a Lévy measure by the so-called Lévy copula after standardizing the marginal Lévy measure to the Lebesgue measure. Contrary to the distributional copula model, which is always a distribution function, the Lévy copula defines a measure which is not a Lévy measure. This approach has the disadvantage that results from [17] connecting regular variation of the

Received 23 March 2009; revision received 29 September 2011.

* Postal address: Centre for Mathematical Sciences, and Institute for Advanced Study, Technische Universität München, 85748 Garching, Germany.

** Email address: cklu@ma.tum.de 
multivariate increment distribution of a Lévy process and its Lévy measure cannot be applied directly.

Anyway, starting from the Lévy measure instead of from the distribution of a Lévy process is often simpler. It guarantees that the corresponding process is a Lévy process. Numerical methods (for instance in finance) are often based on spectral methods, which again require knowledge about the Lévy measure. Also, the statistical assessment of the Lévy measure has made progress recently: Ueltzhöfer and Klüppelberg [27] presented a nonparametric method based on previous work giving ample references, and Esmaeili and Klüppelberg [10]-[12] invoked the Lévy copula idea for parametric estimation.

It was pointed out in Definition 2.2 of [1] that a simple transformation of the one-dimensional marginal Lebesgue measures leads to a Lévy measure with standard 1-stable margins. The resulting dependence function was denoted a Pareto Lévy copula in [21]. This transformation has the advantage that the well-developed theory of multivariate regular variation can be applied to tails of distributions and Lévy measures. In particular, as we will prove, extreme dependence is the same in the Lévy measure and in the distribution of a Lévy process.

The authors of both [1] and [21] restricted their investigations to spectrally positive Lévy processes, i.e. Lévy measures concentrated on the positive cone $[0, \infty)^{d}$. Moreover, their respective focus was rather on applications and not on the understanding and consequences of the marginal transformation. In the present paper we explore this transformation for general Lévy processes in $\mathbb{R}^{d}$ and use the Pareto Lévy measure for a new description of the dependence structure of a Lévy measure. This approach clearly shows how the dependence structure of the Lévy measure affects the extremal behavior (in particular joint large jumps) of the Lévy process in a natural and intuitive way. Furthermore, to quantify the joint extremal jumps of the Lévy process, we introduce tail dependence coefficients $\Lambda_{U}$ and $\Lambda_{\mathrm{L}}$ for the Pareto Lévy measure analogously to such measures for multivariate probability measures.

Our paper is organized as follows. In Section 2 we recall basic knowledge about multivariate regular variation of Lévy measures and formulate Sklar's theorem for Pareto Lévy measures. We also present several examples, including a nonhomogeneous example, which gives rise to a Lévy process with regularly varying marginal Lévy processes, which is not multivariate regularly varying. Our main results about regular variation of a Lévy measure and its Pareto Lévy measure are proved in Section 3. We also introduce upper and lower tail dependence coefficients for the Lévy measure. In Section 4 we calculate the limit measure of a bivariate regularly varying Lévy measure for homogeneous Pareto Lévy measures and also consider the nonhomogeneous example outside multivariate regular variation. For $d=2$, we also show graphical representations of the dependence structure in Section 5.

\section{Preliminaries}

We assume that all random elements considered are defined on a common probability space $(\Omega, \mathcal{F}, \mathrm{P})$. For a topological space $\mathbb{T}$ its Borel $\sigma$-algebra is denoted by $\mathcal{B}(\mathbb{T})$. For $B \in \mathcal{B}(\mathbb{T})$, we denote by $B^{\circ}$ and by $\bar{B}$ the interior and the closure of $B$, respectively, and $\partial B=\bar{B} \backslash B^{\circ}$ is the boundary of $B$.

Regular variation of Lévy measures is formulated in terms of vague convergence of Radon measures on $\mathbb{E}:=[-\infty, \infty]^{d} \backslash\{\boldsymbol{0}\}$, where $\mathbf{0}:=(0, \ldots, 0)$ is the zero in $\mathbb{R}^{d}$, and we also define $\infty:=(\infty, \ldots, \infty)$. The space $\mathbb{E}$ is equipped with the usual topology such that $\mathcal{B}(\mathbb{E})=$ $\mathscr{B}\left(\mathbb{R}^{d}\right) \cap \mathbb{E}$, and the Borel sets of $\mathbb{R}^{d}$ bounded away from $\mathbf{0}$ are relatively compact in $\mathbb{E}$.

For $\boldsymbol{a}, \boldsymbol{b} \in \mathbb{R}^{d}$, we write $\boldsymbol{a}<\boldsymbol{b}$ if this holds componentwise. For $a, b \in \mathbb{R}$, we write $a \vee b:=\max \{a, b\}$. For a set $I$, we define $|I|$ as its cardinality. 
Let $\boldsymbol{X}:=\left(\boldsymbol{X}_{t}\right)_{t \geq 0}$ be a Lévy process in $\mathbb{R}^{d}$ with characteristic triple $(\boldsymbol{\gamma}, A, \Pi)$, where $\gamma \in \mathbb{R}^{d}, A$ is a symmetric nonnegative definite $d \times d$ matrix, and the Lévy measure $\Pi$ is a measure on $\mathbb{R}^{d}$ satisfying $\Pi(\{\mathbf{0}\})=0$ and $\int_{\mathbb{R}^{d}} \min \left\{1,|\boldsymbol{x}|^{2}\right\} \Pi(\mathrm{d} \boldsymbol{x})<\infty$, where $|\cdot|$ denotes an arbitrary norm in $\mathbb{R}^{d}$. The Lévy-Khintchine representation gives $\mathrm{E}\left[\mathrm{e}^{\mathrm{i}\left(\boldsymbol{z}, \boldsymbol{X}_{t}\right)}\right]=\mathrm{e}^{-t \Psi(z)}$ for $z \in \mathbb{R}^{d}$ and $t \geq 0$, where

$$
\Psi(z)=\mathrm{i}(\boldsymbol{\gamma}, \boldsymbol{z})+\frac{1}{2} z^{\top} A z+\int_{\mathbb{R}^{d}}\left(1-\mathrm{e}^{\mathrm{i}(\boldsymbol{z}, \boldsymbol{x})}+\mathrm{i}(\boldsymbol{z}, \boldsymbol{x}) 1_{\{|\boldsymbol{x}| \leq 1\}}\right) \Pi(\mathrm{d} \boldsymbol{x})
$$

and $(\cdot, \cdot)$ denotes the inner product in $\mathbb{R}^{d}$. We consider a Lévy measure $\Pi$ on $\mathbb{E}$ by setting $\Pi(B):=\Pi\left(B \cap \mathbb{R}^{d}\right)$ for $B \in \mathscr{B}(\mathbb{E})$. Furthermore, we assume throughout that the sample paths of $\boldsymbol{X}$ are càdlàg. For details and more background on Lévy processes, we refer the reader to [26].

\subsection{Multivariate regular variation of Lévy measures}

The notion of multivariate regular variation of a random vector has been in the focus of multivariate extreme value theory for years; cf. [23] and [24]. A reformulation of the definition in terms of a multivariate Lévy measure, which in most cases is an infinite measure, requires an extension.

Definition 1. (Regular variation of Lévy measures; cf. [16, Section 3].) A Lévy measure П on $\mathbb{E}$ is called regularly varying if one of the following equivalent definitions holds.

(i) There exists a norming sequence $\left\{c_{n}\right\}_{n \in \mathbb{N}}$ of positive numbers with $c_{n} \uparrow \infty$ as $n \rightarrow \infty$ and a nonzero Radon measure $\mu$ on $\mathscr{B}(\mathbb{E})$ with $\mu\left(\overline{\mathbb{R}}^{d} \backslash \mathbb{R}^{d}\right)=0$ such that

$$
n \Pi\left(c_{n} \cdot\right) \stackrel{\mathrm{v}}{\rightarrow} \mu(\cdot) \quad \text { as } n \rightarrow \infty,
$$

where ' $\stackrel{\mathrm{v}}{\rightarrow}$ ' denotes vague convergence in $\mathscr{B}(\mathbb{E})$. Then necessarily the limit measure $\mu$ is homogeneous of some degree $\alpha>0$ (called the index of regular variation), i.e. $\mu(t \cdot)=$ $t^{-\alpha} \mu(\cdot)$ for all $t>0$. For $\Pi$ regularly varying with index $\alpha$, norming sequence $c_{n}$, and limit measure $\mu$, we will write $\Pi \in \operatorname{RV}\left(\alpha, c_{n}, \mu\right)$.

(ii) There exists a finite nonzero measure $\mu_{\mathbb{S}}$ on $\mathcal{B}(\mathbb{S})$, where $\mathbb{S}:=\left\{\boldsymbol{x} \in \mathbb{R}^{d}:|\boldsymbol{x}|=1\right\}$ denotes the unit sphere with respect to a norm $|\cdot|$ on $\mathbb{R}^{d}$, such that, for all $u>0$,

$$
\frac{\Pi\left(\left\{\boldsymbol{x} \in \mathbb{R}^{d}:|\boldsymbol{x}|>t u, \boldsymbol{x} /|\boldsymbol{x}| \in \cdot\right\}\right)}{\Pi\left(\left\{\boldsymbol{x} \in \mathbb{R}^{d}:|\boldsymbol{x}|>t\right\}\right)} \stackrel{\mathrm{w}}{\rightarrow} u^{-\alpha} \mu_{\mathbb{S}}(\cdot) \quad \text { as } t \rightarrow \infty,
$$

where ' $\stackrel{\mathrm{w}}{\rightarrow}$ ' denotes weak convergence in $\mathcal{B}(\mathbb{S})$. We call $\mu_{\mathbb{S}}$ the spectral measure of $\Pi$.

\subsection{The Pareto Lévy measure}

We use the same notation as in [20]. Define $\operatorname{sgn}(x):=1_{\{x \geq 0\}}-1_{\{x<0\}}$ and

$$
\mathscr{I}(x):= \begin{cases}(x, \infty), & x \geq 0 \\ (-\infty, x], & x<0 .\end{cases}
$$

Definition 2. (Tail integral of a Lévy measure.) Let $\boldsymbol{X}$ be a Lévy process in $\mathbb{R}^{d}$ with Lévy measure $\Pi$. The tail integral of $\boldsymbol{X}$ is the function $\bar{\Pi}:(\overline{\mathbb{R}} \backslash\{0\})^{d} \rightarrow \mathbb{R}$ defined as

$$
\bar{\Pi}\left(x_{1}, \ldots, x_{d}\right):=\Pi\left(\prod_{i=1}^{d} \tau\left(x_{i}\right)\right) \prod_{j=1}^{d} \operatorname{sgn}\left(x_{j}\right) .
$$


By (1), all tail integrals are right-continuous functions on $(\mathbb{R} \backslash\{0\})^{d}$. The tail integral does not determine the Lévy measure uniquely, because it does not specify its mass on $\mathbb{R}^{d} \backslash(\mathbb{R} \backslash\{0\})^{d}$.

Definition 3. (Margins of a Lévy process/Lévy measure/tail integral.) Let

$$
\boldsymbol{X}=\left(X^{1}, \ldots, X^{d}\right)=\left(X_{t}^{1}, \ldots, X_{t}^{d}\right)_{t \geq 0}
$$

be a Lévy process in $\mathbb{R}^{d}$ with Lévy measure $\Pi$ and $I \subseteq\{1, \ldots, d\}$ a nonempty index set. We define the following quantities.

(i) The $I$-margin of $X$ is the Lévy process $X^{I}:=\left(X^{i}\right)_{i \in I}$.

(ii) Let $\Pi_{I}$ be the Lévy measure of $X^{I}$, denoted the $I$-marginal Lévy measure. Then

$$
\Pi_{I}(A)=\Pi\left(\left\{\boldsymbol{x} \in \mathbb{R}^{d}:\left(x_{i}\right)_{i \in I} \in A\right\}\right), \quad A \in \mathcal{B}\left(\mathbb{R}^{|I|} \backslash\{\mathbf{0}\}\right) .
$$

(iii) The I-marginal tail integral of $\boldsymbol{X}$ is given by $\bar{\Pi}_{I}:(\overline{\mathbb{R}} \backslash\{0\})^{|I|} \rightarrow \mathbb{R}$ with

$$
\bar{\Pi}_{I}\left(\left(x_{i}\right)_{i \in I}\right)=\Pi_{I}\left(\prod_{i \in I} \mathcal{I}\left(x_{i}\right)\right) \prod_{i \in I} \operatorname{sgn}\left(x_{i}\right) .
$$

To simplify notation, we denote one-dimensional margins by $X^{i}, \Pi_{i}$, and $\bar{\Pi}_{i}$.

By [20, Lemma 3.5], the set of all marginal tail integrals $\left\{\bar{\Pi}_{I}: I \subseteq\{1, \ldots, d\}\right\}$ determines the Lévy measure $\Pi$ uniquely and vice versa.

The following lemma is well known; a proof can be found, for instance, in [8, Lemma 2.0.2].

Lemma 1. Let $\Pi$ be a d-dimensional Lévy measure. If $\Pi \in \operatorname{RV}\left(\alpha, c_{n}, \mu\right)$ then, for $x>0$ and all $i=1, \ldots, d$,

$$
n \bar{\Pi}_{i}\left(c_{n} x\right) \rightarrow \bar{\mu}_{i}(1) x^{-\alpha} \text { and } n \bar{\Pi}_{i}\left(-c_{n} x\right) \rightarrow \bar{\mu}_{i}(-1) x^{-\alpha} \text { as } n \rightarrow \infty,
$$

where $\mu_{i}(B):=\mu\left(\left\{\boldsymbol{x} \in \mathbb{E}: x_{i} \in B\right\}\right)$ for $B \in \mathcal{B}(\overline{\mathbb{R}} \backslash\{0\})$ and $\bar{\mu}_{i}(1),\left|\bar{\mu}_{i}(-1)\right| \in[0, \infty)$.

Furthermore, there exists an index $i_{*} \in\{1, \ldots, d\}$ such that $\bar{\mu}_{i_{*}}(1)-\bar{\mu}_{i_{*}}(-1)>0$ and $\Pi_{i_{*}} \in \mathrm{RV}\left(\alpha, c_{n}, \mu_{i_{*}}\right)$.

Now we present our reference Lévy measure; it has been proposed in [21].

Definition 4. (Pareto Lévy measure, Pareto Lévy copula.) Let $\Gamma$ be a $d$-dimensional Lévy measure with one-dimensional marginals $\Gamma_{i}\left(\mathrm{~d} x_{i}\right)=\left|x_{i}\right|^{-2} \mathrm{~d} x_{i}$ on $\mathbb{R} \backslash\{0\}$. Then we call $\Gamma$ the Pareto Lévy measure (PLM) and its tail integral $\bar{\Gamma}$ is called the Pareto Lévy copula (PLC).

The margins $\Gamma_{i}$ are Lévy measures of 1-stable Lévy processes, but $\Gamma$ is in general not the Lévy measure of a 1-stable Lévy process.

The following result has been proved for Lévy copulas in [20, Theorem 3.6]. For a detailed proof of this transformed version, we refer the reader to [8, Theorem 1.1.10].

Theorem 1. (Sklar's theorem for Pareto Lévy measures.) (i) Let $\boldsymbol{X}$ be a Lévy process in $\mathbb{R}^{d}$ with Lévy measure $\Pi$. Let $\varnothing \neq I \subseteq\{1, \ldots, d\}$ be an arbitrary index set. Then there exists a PLM $\Gamma$ such that

$$
\bar{\Pi}_{I}\left(\left(x_{i}\right)_{i \in I}\right)=\bar{\Gamma}_{I}\left(\left(\frac{1}{\bar{\Pi}_{i}\left(x_{i}\right)}\right)_{i \in I}\right), \quad\left(x_{i}\right)_{i \in I} \in(\overline{\mathbb{R}} \backslash\{0\})^{|I|} .
$$

The PLM $\Gamma$ is unique on $\prod_{i=1}^{d} \overline{\operatorname{Ran}\left(1 / \bar{\Pi}_{i}\right)}$. 
(ii) Let $\Gamma$ be a d-dimensional PLM, and let $\bar{\Pi}_{i}$ for $i=1, \ldots, d$ be one-dimensional tail integrals of arbitrary Lévy processes. Then there exists a Lévy process $\boldsymbol{X}$ in $\mathbb{R}^{d}$, whose components have tail integrals $\bar{\Pi}_{1}, \ldots, \bar{\Pi}_{d}$ and whose marginal Lévy measures satisfy(2) for every nonempty $I \subseteq$ $\{1, \ldots, d\}$ and every $\left(x_{i}\right)_{i \in I} \in(\overline{\mathbb{R}} \backslash\{0\})^{|I|}$. The Lévy measure $\Pi$ of $\boldsymbol{X}$ is uniquely determined by $\Gamma$ and $\bar{\Pi}_{1}, \ldots, \bar{\Pi}_{d}$.

Remark 1. (Relation between Lévy copula and Pareto Lévy measure.) (i) Every Lévy copula $\hat{C}$ uniquely defines a PLM $\Gamma$, given for $\boldsymbol{x}, \boldsymbol{y} \in \mathbb{R}^{d}$ such that $\mathbf{0} \notin[\boldsymbol{x}, \boldsymbol{y})$ by

$$
\Gamma([\boldsymbol{x}, \boldsymbol{y}))=\sum_{\boldsymbol{u} \in\left\{1 / y_{1}, 1 / x_{1}\right\} \times \cdots \times\left\{1 / y_{d}, 1 / x_{d}\right\}}(-1)^{N(\boldsymbol{u})} \hat{\boldsymbol{C}}(\boldsymbol{u}),
$$

where $\boldsymbol{u}=\left(u_{1}, \ldots, u_{d}\right) \in(-\infty, \infty]^{d}, N(\boldsymbol{u}):=\#\left\{k: u_{k}=1 / y_{k}\right\}$, and $1 / 0:=\infty$. Furthermore, for the PLC $\bar{\Gamma}$, we have, for $\boldsymbol{x}=\left(x_{1}, \ldots, x_{d}\right) \in(\overline{\mathbb{R}} \backslash\{0\})^{d}$,

$$
\bar{\Gamma}\left(x_{1}, \ldots, x_{d}\right)=\hat{C}\left(\frac{1}{x_{1}}, \ldots, \frac{1}{x_{d}}\right) .
$$

Consequently, for a PLM $\Gamma$ with Lévy copula $\hat{C}$ the following statements are equivalent.

1. $\Gamma$ is the Lévy measure of a 1-stable Lévy process.

2. $\Gamma$ is homogeneous of degree 1, i.e. $\Gamma(t A)=t^{-1} \Gamma(A)$ for all $t>0$ and $A \in \mathscr{B}(\mathbb{E})$.

3. $\hat{C}$ satisfies, for all $t>0$,

$$
\hat{C}\left(t x_{1}, \ldots, t x_{d}\right)=t \hat{C}\left(x_{1}, \ldots, x_{d}\right), \quad x \in \mathbb{R}^{d} .
$$

(ii) From part (i) we immediately identify the Lévy copula from [20].

The advantage of working with a Pareto Lévy measure instead of a Lévy copula should be clear: the Pareto Lévy measure is always a Lévy measure. We will see below that this is the appropriate framework for regularly varying Lévy processes.

Since Lévy measures can be singular at the origin, the Lévy measure on the hyperplanes through the axes needs special attention. Furthermore, since PLCs are defined quadrantwise, special care has to be taken for sets which are not concentrated in one single quadrant. The following result presents the Lévy measure $\Pi$ of arbitrary rectangles in terms of the PLM $\Gamma$ and the marginal tail integrals $\bar{\Pi}_{i}$ for $i=1, \ldots, d$. For the proof, see the proof of Proposition 1.1.11 of [8, Appendix].

Proposition 1. Let $\Gamma$ be a PLM, and let $\Pi_{i}$ for $i=1, \ldots, d$ be one-dimensional Lévy measures. Let $\Pi$ be the Lévy measure defined by (2). With $\bar{\Pi}_{i}(0):=\bar{\Pi}_{i}(0+)=\lim _{x \downarrow} \bar{\Pi}_{i}(x)$ and $\bar{\Pi}_{i}(0-)=\lim _{x \uparrow 0} \bar{\Pi}_{i}(x)$, the following assertions hold.

(i) For $\boldsymbol{a}, \boldsymbol{b} \in \mathbb{R}^{d}$ with $\mathbf{0} \notin \prod_{i=1}^{d}\left(a_{i}, b_{i}\right]$,

$$
\Pi\left(\prod_{i=1}^{d}\left(a_{i}, b_{i}\right]\right)=\Gamma\left(\prod_{i=1}^{d}\left(\frac{1}{\bar{\Pi}_{i}\left(a_{i}\right)}, \frac{1}{\bar{\Pi}_{i}\left(b_{i}\right)}\right]\right) .
$$


(ii) Let $\varnothing \neq K \subset\{1, \ldots, d\}$. Define

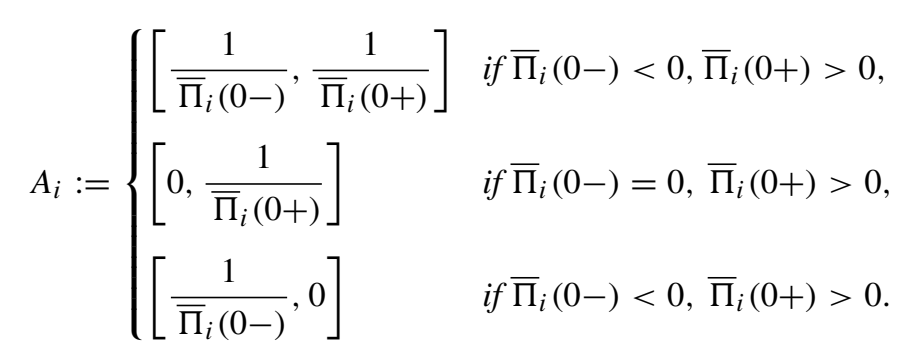

For $\boldsymbol{a}, \boldsymbol{b} \in \mathbb{R}^{d}$ with $\mathbf{0} \notin \prod_{i \in K}\{0\} \times \prod_{i \notin K}\left(a_{i}, b_{i}\right]$,

$$
\Pi\left(\prod_{i \in K}\{0\} \times \prod_{i \notin K}\left(a_{i}, b_{i}\right]\right)=\Gamma\left(\prod_{i \in K} A_{i} \times \prod_{i \notin K}\left(\frac{1}{\bar{\Pi}_{i}\left(a_{i}\right)}, \frac{1}{\bar{\Pi}_{i}\left(b_{i}\right)}\right]\right) .
$$

Conversely, the PLM $\Gamma$ that was constructed from $\Pi$ to satisfy (2) can be given for arbitrary rectangles in terms of the Lévy measure $\Pi$ and its marginal tail integrals $\bar{\Pi}_{i}$ for $i=1, \ldots, d$. It follows directly from the construction of the measure $m$ and the Lévy copula in the proof of Sklar's theorem in [20] with the transformation of the marginals to 1-stable instead of Lebesgue measures.

Define, for $x \in(-\infty, \infty]$ and $i=1, \ldots, d$, the extended marginals $\dot{\bar{\Pi}}_{i}(x):=\bar{\Pi}_{i}(x)$ for $x \neq 0$ and $\infty$ for $x=0$ (which holds for all processes apart from compound Poisson processes anyway). Define also

$$
\Delta \bar{\Pi}_{i}(x):= \begin{cases}\lim _{\xi \uparrow x} \bar{\Pi}_{i}(\xi)-\bar{\Pi}_{i}(x)=\Pi_{i}(\{x\}) & \text { for } x \neq 0 \\ 0 & \text { for } x=0 .\end{cases}
$$

Denote by $\lambda$ the Lebesgue measure on $\mathbb{R}$ and by $\delta_{\boldsymbol{x}}$ the Dirac measure in $\boldsymbol{x}$.

Proposition 2. Let $\Pi$ be a Lévy measure with one-dimensional margins $\Pi_{i}$ for $i=1, \ldots, d$. Define

$$
\mathscr{D}_{i}:=\mathcal{I}\left(\frac{1}{\bar{\Pi}_{i}(0-)}\right) \cup \mathcal{I}\left(\frac{1}{\bar{\Pi}_{i}(0+)}\right) \cup\{0\} .
$$

For the PLM $\Gamma$ constructed from $\Pi$ as in Theorem 1(i), the following assertions hold.

(i) For $\boldsymbol{a}, \boldsymbol{b} \in \mathbb{R}^{d}$ with $(\boldsymbol{a}, \boldsymbol{b}] \subset \prod_{i=1}^{d} \mathcal{D}_{i}$,

$$
\begin{aligned}
& \Gamma((\boldsymbol{a}, \boldsymbol{b}])=\left.\Pi \otimes \lambda\right|_{[0,1]^{d}}(\left\{\left(x_{1}, \ldots, x_{d}, y_{1}, \ldots, y_{d}\right) \in\left(\mathbb{R}^{d} \backslash\{\mathbf{0}\}\right) \times[0,1]^{d}:\right. \\
&\left.\left.\frac{1}{\dot{\bar{\Pi}}_{i}\left(x_{i}\right)+y_{i} \Delta \bar{\Pi}_{i}\left(x_{i}\right)} \in\left(a_{i}, b_{i}\right] \text { for } i=1, \ldots, d\right\}\right) .
\end{aligned}
$$

(ii) For $\boldsymbol{a}, \boldsymbol{b} \in \mathbb{R}^{d}$ with $(\boldsymbol{a}, \boldsymbol{b}] \subset \mathbb{R}^{d} \backslash \prod_{i=1}^{d} \mathscr{D}_{i}$,

$$
\Gamma((\boldsymbol{a}, \boldsymbol{b}])=\sum_{i=1}^{d} \underbrace{\delta_{0} \otimes \cdots \otimes \delta_{0}}_{i-1} \otimes \Gamma_{i} \otimes \underbrace{\delta_{0} \otimes \cdots \otimes \delta_{0}}_{d-i}((\boldsymbol{a}, \boldsymbol{b}]),
$$

where $\Gamma_{i}\left(\mathrm{~d} x_{i}\right)=\left|x_{i}\right|^{-2} \mathrm{~d} x_{i}$ for $x_{i} \in \mathbb{R} \backslash\{0\}$. 
Remark 2. For a multivariate compound Poisson process, the sets $\mathscr{D}_{i}$ are equal to

$$
\mathscr{D}_{i}=\left(-\infty,-\frac{1}{\lambda_{i}^{-}}\right] \cup\left(\frac{1}{\lambda_{i}^{+}}, \infty\right) \cup\{0\}
$$

for $i=1, \ldots, d$, where $\lambda_{i}^{-}, \lambda_{i}^{+}>0$ are the intensities of the positive and negative Poisson processes, respectively. The above construction in Proposition 2 ensures that the resulting PLM has indeed 1-stable margins.

Example 1. (Independence PLM.) The jumps of a Lévy process are independent, if the Lévy measure is supported by the coordinate axes, i.e. the independence PLM is given by

$$
\Gamma_{\perp}(A)=\sum_{i=1}^{d} \Gamma_{i}\left(A_{i}\right) \quad \text { for } A \in \mathcal{B}\left(\mathbb{R}^{d} \backslash\{\mathbf{0}\}\right),
$$

where $A_{i}:=\left\{x_{i} \in \mathbb{R}:\left(0, \ldots, 0, x_{i}, 0, \ldots, 0\right) \in A\right\}$ and $\Gamma_{i}$ denotes the Lévy measure of a one-dimensional standard 1-stable Lévy process. By [20, Lemma 3.5], $\Gamma$ is characterized by the marginal tail integrals $\left(\bar{\Gamma}_{\perp, I}\right)_{I \subseteq\{1, \ldots, d\}}$, given for $\left(x_{1}, \ldots, x_{|I|}\right) \in(\overline{\mathbb{R}} \backslash\{0\})^{|I|}$ by

$$
\bar{\Gamma}_{\perp, I}\left(x_{1}, \ldots, x_{|I|}\right)= \begin{cases}0 & \text { if }|I|>1 \\ x^{-1} & \text { if }|I|=1\end{cases}
$$

Example 2. (Complete positive dependence PLM.) The jumps of a Lévy process are completely dependent or comonotonic, if there exists a strictly ordered set $S \subset K:=\left\{x \in \mathbb{R}^{d}: \operatorname{sgn}\left(x_{1}\right)=\right.$ $\left.\cdots=\operatorname{sgn}\left(x_{d}\right)\right\}$ such that, for almost all sample paths, $\Delta \boldsymbol{X}_{t} \in S$ for $t>0$; see [20, Definition 4.2]. In this case, all components jump almost surely together and, therefore, the PLM of complete positive dependence is concentrated on $(\mathbb{R} \backslash\{0\})^{d}$. So $\Gamma$ is characterized by the corresponding PLC, given for $\left(x_{1}, \ldots, x_{d}\right) \in(\overline{\mathbb{R}} \backslash\{0\})^{d}$ by

$$
\bar{\Gamma}_{||}\left(x_{1}, \ldots, x_{d}\right)=\frac{1}{\left|x_{1}\right| \vee \cdots \vee\left|x_{d}\right|} 1_{K}\left(\left(x_{1}, \ldots, x_{d}\right)\right) \prod_{j=1}^{d} \operatorname{sgn}\left(x_{j}\right)
$$

and supported by $\left\{\boldsymbol{x} \in \mathbb{R}^{d} \backslash\{\boldsymbol{0}\}: x_{1}=\cdots=x_{d}\right\}$.

Example 3. (Archimedean PLM.) Analogously to the Archimedean copula construction (cf. [22, Section 4]), Archimedean Pareto Lévy measures can be defined by constructing their PLC on $(\mathbb{R} \backslash\{0\})^{d}$ and setting $\Gamma\left(\mathbb{R}^{d} \backslash(\mathbb{R} \backslash\{0\})^{d}\right)=0$. Let $\varphi:[-1,1] \rightarrow[-\infty, \infty]$ be a strictly increasing continuous function with $\varphi(1)=\infty, \varphi(0)=0$, and $\varphi(-1)=-\infty$, having derivatives of order up to $d$ on $(-1,0)$ and $(0,1)$, satisfying, for all $k=1, \ldots, d$,

$$
\frac{\partial^{k} \varphi(u)}{\partial u^{k}} \geq 0, \quad u \in(0,1), \quad \text { and } \quad(-1)^{k} \frac{\partial^{k} \varphi(u)}{\partial u^{k}} \leq 0, \quad u \in(-1,0) .
$$

Set $\tilde{\varphi}(u):=2^{d-2}(\varphi(u)-\varphi(-u))$ for $u \in[-1,1]$. Then

$$
\bar{\Gamma}\left(x_{1}, \ldots, x_{d}\right)=\varphi\left(\prod_{i=1}^{d} \tilde{\varphi}^{-1}\left(\frac{1}{x_{i}}\right)\right), \quad\left(x_{1}, \ldots, x_{d}\right) \in(\overline{\mathbb{R}} \backslash\{0\})^{d},
$$

is a PLC; see [20, Theorem 6.1]. If we construct a Lévy measure $\Pi$ by margins $\Pi_{i}$ for $i=1, \ldots, d$ and an Archimedean PLM, $\Pi$ may have mass on $\mathbb{R}^{d} \backslash(\mathbb{R} \backslash\{0\})^{d}$, although $\Gamma$ does not. From (4) we see that $\Pi\left(\mathbb{R}^{d} \backslash(\mathbb{R} \backslash\{0\})^{d}\right)>0$ if and only if $\bar{\Pi}_{i}(0+)<\infty$ or $\bar{\Pi}_{i}(0-)>-\infty$ for at least one $i$. 
Example 4. (Clayton PLM.) Setting

$$
\varphi(x)=(-\log |x|)^{-1 / \theta}\left(\eta 1_{\{x>0\}}-(1-\eta) 1_{\{x<0\}}\right), \quad \theta>0, \eta \in(0,1),
$$

in Example 3 corresponds to the Clayton Pareto Lévy measure, and the Clayton Pareto Lévy copula is, for $\theta>0$ and $\eta \in[0,1]$, given for $\left(x_{1}, \ldots, x_{d}\right) \in(\overline{\mathbb{R}} \backslash\{0\})^{d}$ by

$$
\bar{\Gamma}_{\eta, \theta}\left(x_{1}, \ldots, x_{d}\right)=2^{2-d}\left(\sum_{i=1}^{d}\left|x_{i}\right|^{\theta}\right)^{-1 / \theta}\left(\eta 1_{\left\{x_{1} \cdots x_{d}>0\right\}}-(1-\eta) 1_{\left\{x_{1} \cdots x_{d}<0\right\}}\right) .
$$

For $d=2$, this reduces to

$$
\bar{\Gamma}_{\eta, \theta}\left(x_{1}, x_{2}\right)=\left(\left|x_{1}\right|^{\theta}+\left|x_{2}\right|^{\theta}\right)^{-1 / \theta}\left(\eta 1_{\left\{x_{1} x_{2}>0\right\}}-(1-\eta) 1_{\left\{x_{1} x_{2}<0\right\}}\right),
$$

which covers with its single parameter $\theta$ the whole range of dependence; see, e.g. [4], [5], and [9] for applications in insurance and operational risk modeling. This PLC has also been the focus of parametric estimation in [10], [11], and [12]. The Clayton PLM is homogeneous of degree 1 .

Example 5. (Nonhomogeneous PLM.) Setting

$$
\varphi(x)=\zeta \frac{|x|}{1-|x|}\left(\eta 1_{\{x>0\}}-(1-\eta) 1_{\{x<0\}}\right), \quad \zeta>0, \eta \in(0,1),
$$

in Example 3 yields the PLC for $\zeta>0$ and $\eta \in[0,1]$ given for $\left(x_{1}, \ldots, x_{d}\right) \in(\overline{\mathbb{R}} \backslash\{0\})^{d}$ as

$$
\bar{\Gamma}_{\eta, \zeta}\left(x_{1}, \ldots, x_{d}\right)=\frac{\zeta \prod_{i=1}^{d}\left|1 / x_{i}\right|}{\prod_{i=1}^{d}\left(\left|1 / x_{i}\right|+\zeta\right)-\prod_{i=1}^{d}\left|1 / x_{i}\right|}\left(\eta 1_{\left\{x_{1} \cdots x_{d}>0\right\}}-(1-\eta) 1_{\left\{x_{1} \cdots x_{d}<0\right\}}\right) .
$$

For $d=2$, the PLC $\bar{\Gamma}_{\eta, \zeta}$ reduces to

$$
\bar{\Gamma}_{\eta, \zeta}\left(x_{1}, x_{2}\right)=\frac{1}{\left|x_{1}\right|+\left|x_{2}\right|+\zeta\left|x_{1} x_{2}\right|}\left(\eta 1_{\left\{x_{1} x_{2}>0\right\}}-(1-\eta) 1_{\left\{x_{1} x_{2}<0\right\}}\right),
$$

which was treated in [9, Example 2.8(d)]. Obviously, this PLM has homogeneous margins, but is not homogeneous of degree 1 . Consequently, the corresponding Lévy process has regularly varying marginal processes, but is not regularly varying.

\section{Regular variation and the Pareto Lévy copula}

As stated in Lemma 1, multivariate regular variation of $\Pi$ implies regular variation of at least one of the one-dimensional marginal Lévy measures $\Pi_{i}$. To prove the converse, we assume without loss of generality that $\Pi_{1} \in \operatorname{RV}\left(\alpha, c_{n}, \mu_{1}\right)$. We also assume that the following tail balance conditions hold for $x>0$ and all $i=1, \ldots, d$ :

$$
\lim _{n \rightarrow \infty} n \bar{\Pi}_{i}\left(c_{n} x\right)=p_{i}^{+} x^{-\alpha} \text { and } \lim _{n \rightarrow \infty}-n \bar{\Pi}_{i}\left(-c_{n} x\right)=p_{i}^{-} x^{-\alpha},
$$

where $p_{i}^{+}, p_{i}^{-} \in[0, \infty)$. For $x \in \overline{\mathbb{R}}$, we define

$$
p_{i}^{\operatorname{sgn}(x)}:= \begin{cases}p_{i}^{+} & \text {if } x \geq 0 \\ p_{i}^{-} & \text {if } x<0\end{cases}
$$

The following result has been proved for copulas in Theorem 3.1 of [21]. 
Theorem 2. Let $\Gamma$ be a PLM, and let $\Pi_{i}$ for $i=1, \ldots, d$ be one-dimensional Lévy measures. Let $\Pi$ be the d-dimensional Lévy measure defined in (3). Suppose that $\Pi_{1} \in \operatorname{RV}\left(\alpha, c_{n}, \mu_{1}\right)$ and that the tail balance conditions (9) for the margins hold. Furthermore, suppose that $\Gamma \in \operatorname{RV}(1, n, v)$. Then $\Pi \in \operatorname{RV}\left(\alpha, c_{n}, \mu\right)$, where $\mu$ is given by

$$
\mu((\boldsymbol{a}, \boldsymbol{b}])=v((\tilde{\boldsymbol{a}}, \tilde{\boldsymbol{b}}]), \quad \boldsymbol{a}, \boldsymbol{b} \in \mathbb{R}^{d},
$$

and $\tilde{\boldsymbol{a}}=\left(\tilde{a}_{i}\right)_{i=1, \ldots, d}$ is given by

$$
\tilde{a}_{i}:= \begin{cases}0 & \text { if } a_{i}=0, \\ \operatorname{sgn}\left(a_{i}\right)\left(p_{i}^{\operatorname{sgn}\left(a_{i}\right)}\right)^{-1}\left|a_{i}\right|^{\alpha} & \text { if } a_{i} \neq 0, p_{i}^{\operatorname{sgn}\left(a_{i}\right)}>0, \\ \infty & \text { if } a_{i}>0, p_{i}^{\operatorname{sgn}\left(a_{i}\right)}=0, \\ -\infty & \text { if } a_{i}<0, p_{i}^{\operatorname{sgn}\left(a_{i}\right)}=0,\end{cases}
$$

with $\tilde{\boldsymbol{b}}=\left(\tilde{b}_{i}\right)_{i=1, \ldots, d}$ defined analogously.

Proof. First we show that $\left\{n \Pi\left(c_{n} \cdot\right)\right\}_{n \in \mathbb{N}}$ is relatively compact in the vague topology. Since $\Pi$ is a Lévy measure, for the ball $B_{\mathbf{0}, r}=\left\{\boldsymbol{x} \in \mathbb{R}^{d}:|\boldsymbol{x}-\mathbf{0}|<r\right\}$, we obtain

$$
\sup _{n \in \mathbb{N}} n \Pi\left(c_{n}\left(\mathbb{R}^{d} \backslash B_{0, r}\right)\right)<\infty \text { for all } r>0,
$$

and, by [19, Theorem 15.7.5], the sequence $\left\{n \Pi\left(c_{n} \cdot\right)\right\}_{n \in \mathbb{N}}$ is relatively compact. So there are subsequential vague limits and, by [16, Theorem 2.8], we have to show convergence for sets in a determining class. The sets $\{(\boldsymbol{a}, \boldsymbol{b}]: \boldsymbol{a}, \boldsymbol{b} \in \mathbb{E}, \boldsymbol{a} \leq \boldsymbol{b}\}$ are a $\cap$-stable generator of $\mathcal{B}(\mathbb{E})$, but, since $\Pi\left(\overline{\mathbb{R}}^{d} \backslash \mathbb{R}^{d}\right)=0$, it is sufficient to investigate convergence on the sets $\{(\boldsymbol{a}, \boldsymbol{b}]: \boldsymbol{a}, \boldsymbol{b} \in$ $\left.\mathbb{R}^{d} \backslash\{\boldsymbol{0}\}, \boldsymbol{a} \leq \boldsymbol{b}\right\}$. Consequently, we have to show that $\lim _{n \rightarrow \infty} n \Pi\left(c_{n}(\boldsymbol{a}, \boldsymbol{b}]\right)=\mu((\boldsymbol{a}, \boldsymbol{b}])$ for all sets $(\boldsymbol{a}, \boldsymbol{b}]$ with $\boldsymbol{a}, \boldsymbol{b} \in \mathbb{R}^{d} \backslash\{\mathbf{0}\}, \mathbf{0} \notin \overline{(\boldsymbol{a}, \boldsymbol{b}]}$, and

$$
\mu(\partial(\boldsymbol{a}, \boldsymbol{b}])=\mu\left(\bigcup_{k=1}^{d} \prod_{i<k}\left[a_{i}, b_{i}\right] \times\left\{a_{k}, b_{k}\right\} \times \prod_{i>k}\left[a_{i}, b_{i}\right]\right)=0,
$$

where $\mu$ is a nonzero Radon measure with $\mu\left(\overline{\mathbb{R}}^{d} \backslash\{\mathbf{0}\}\right)=0$ and homogeneous of degree $\alpha$. For $\boldsymbol{a}, \boldsymbol{b} \in \mathbb{E}$ and the weight constants $p_{i}$ given in (9), we define the index sets

$$
\begin{aligned}
K_{1} & :=\left\{i: a_{i} b_{i} \neq 0, p_{i}^{\operatorname{sgn}\left(a_{i}\right)} p_{i}^{\operatorname{sgn}\left(b_{i}\right)}>0\right\}, \\
K_{2} & :=\left\{i: a_{i} b_{i} \neq 0, p_{i}^{\operatorname{sgn}\left(a_{i}\right)}>0, p_{i}^{\operatorname{sgn}\left(b_{i}\right)}=0\right\}, \\
K_{3} & :=\left\{i: a_{i} b_{i} \neq 0, p_{i}^{\operatorname{sgn}\left(a_{i}\right)}=0, p_{i}^{\operatorname{sgn}\left(b_{i}\right)}>0\right\}, \\
K_{4} & :=\left\{i: a_{i} b_{i}>0, p_{i}^{\operatorname{sgn}\left(a_{i}\right)}=p_{i}^{\operatorname{sgn}\left(b_{i}\right)}=0\right\}, \\
K_{5} & :=\left\{i: a_{i}<0<b_{i}, p_{i}^{\operatorname{sgn}\left(a_{i}\right)}=p_{i}^{\operatorname{sgn}\left(b_{i}\right)}=0\right\}, \\
K_{6} & :=\left\{i: a_{i}=0, p_{i}^{\operatorname{sgn}\left(b_{i}\right)}>0\right\}, \\
K_{7} & :=\left\{i: a_{i}=0, p_{i}^{\operatorname{sgn}\left(b_{i}\right)}=0\right\}, \\
K_{8} & :=\left\{i: b_{i}=0, p_{i}^{\operatorname{sgn}\left(a_{i}\right)}>0\right\}, \\
K_{9} & :=\left\{i: b_{i}=0, p_{i}^{\operatorname{sgn}\left(a_{i}\right)}=0\right\} .
\end{aligned}
$$


Moreover, we set, for $\boldsymbol{a}, \boldsymbol{b} \in \mathbb{E}$ with $\mathbf{0} \notin \overline{(\boldsymbol{a}, \boldsymbol{b}]}$,

$$
\begin{aligned}
& \mu((\boldsymbol{a}, \boldsymbol{b}]):=v\left(\prod_{i \in K_{1}}\left(\frac{\operatorname{sgn}\left(a_{i}\right)}{p_{i}^{\operatorname{sgn}\left(a_{i}\right)}}\left|a_{i}\right|^{\alpha}, \frac{\operatorname{sgn}\left(b_{i}\right)}{p_{i}^{\operatorname{sgn}\left(b_{i}\right)}}\left|b_{i}\right|^{\alpha}\right] \times \prod_{i \in K_{2}}\left(\frac{\operatorname{sgn}\left(a_{i}\right)}{p_{i}^{\operatorname{sgn}\left(a_{i}\right)}}\left|a_{i}\right|^{\alpha}, \infty\right)\right. \\
& \times \prod_{i \in K_{3}}\left(-\infty, \frac{\operatorname{sgn}\left(b_{i}\right)}{p_{i}^{\operatorname{sgn}\left(b_{i}\right)}}\left|b_{i}\right|^{\alpha}\right] \times \prod_{i \in K_{4}} \varnothing \times \prod_{i \in K_{5}}(-\infty, \infty) \\
& \times \prod_{i \in K_{6}}\left(0, \frac{\operatorname{sgn}\left(b_{i}\right)}{p_{i}^{\operatorname{sgn}\left(b_{i}\right)}}\left|b_{i}\right|^{\alpha}\right] \times \prod_{i \in K_{7}}(0, \infty) \\
& \left.\times \prod_{i \in K_{8}}\left(\frac{\operatorname{sgn}\left(a_{i}\right)}{p_{i}^{\operatorname{sgn}\left(a_{i}\right)}}\left|a_{i}\right|^{\alpha}, 0\right] \times \prod_{i \in K_{9}}(-\infty, 0]\right) .
\end{aligned}
$$

Consider sets $(\boldsymbol{a}, \boldsymbol{b}]$ with $\boldsymbol{a}, \boldsymbol{b} \in \mathbb{R}^{d} \backslash\{\boldsymbol{0}\}, \mathbf{0} \notin \overline{(\boldsymbol{a}, \boldsymbol{b}]}$, and $\mu(\partial(\boldsymbol{a}, \boldsymbol{b}])=0$. From relation (3) we obtain

$$
\begin{aligned}
n \Pi\left(c_{n}(\boldsymbol{a}, \boldsymbol{b}]\right)=n \Gamma & n \prod_{i \in K_{1}, K_{2}, K_{3}, K_{4}, K_{5}}\left(\frac{1}{n \bar{\Pi}_{i}\left(c_{n} a_{i}\right)}, \frac{1}{n \bar{\Pi}_{i}\left(c_{n} b_{i}\right)}\right] \\
& \times \prod_{i \in K_{6}, K_{7}}\left(\frac{1}{n \bar{\Pi}_{i}(0+)}, \frac{1}{n \bar{\Pi}_{i}\left(c_{n} b_{i}\right)}\right] \\
& \left.\times \prod_{i \in K_{8}, K_{9}}\left(\frac{1}{n \bar{\Pi}_{i}\left(c_{n} a_{i}\right)}, \frac{1}{n \bar{\Pi}_{i}(0+)}\right]\right) .
\end{aligned}
$$

From the definition of the $p_{i}$ in (9) we conclude that, as $n \rightarrow \infty$,

$$
\begin{aligned}
& \left(\frac{1}{n \bar{\Pi}_{i}\left(c_{n} a_{i}\right)}, \frac{1}{n \bar{\Pi}_{i}\left(c_{n} b_{i}\right)}\right] \rightarrow\left(\frac{\operatorname{sgn}\left(a_{i}\right)}{p_{i}^{\operatorname{sgn}\left(a_{i}\right)}}\left|a_{i}\right|^{\alpha}, \frac{\operatorname{sgn}\left(b_{i}\right)}{p_{i}^{\operatorname{sgn}\left(b_{i}\right)}}\left|b_{i}\right|^{\alpha}\right]=: B_{1} \quad \text { for } i \in K_{1}, \\
& \left(\frac{1}{n \bar{\Pi}_{i}\left(c_{n} a_{i}\right)}, \frac{1}{n \bar{\Pi}_{i}\left(c_{n} b_{i}\right)}\right] \rightarrow\left(\frac{\operatorname{sgn}\left(a_{i}\right)}{p_{i}^{\operatorname{sgn}\left(a_{i}\right)}}\left|a_{i}\right|^{\alpha}, \infty\right):=B_{2} \quad \text { for } i \in K_{2}, \\
& \left(\frac{1}{n \bar{\Pi}_{i}\left(c_{n} a_{i}\right)}, \frac{1}{n \bar{\Pi}_{i}\left(c_{n} b_{i}\right)}\right] \rightarrow\left(-\infty, \frac{\operatorname{sgn}\left(b_{i}\right)}{p_{i}^{\operatorname{sgn}\left(b_{i}\right)}}\left|b_{i}\right|^{\alpha}\right]=: B_{3} \quad \text { for } i \in K_{3} \text {, } \\
& \left(\frac{1}{n \bar{\Pi}_{i}\left(c_{n} a_{i}\right)}, \frac{1}{n \bar{\Pi}_{i}\left(c_{n} b_{i}\right)}\right] \rightarrow \varnothing=: B_{4} \quad \text { for } i \in K_{4} \text {, } \\
& \left(\frac{1}{n \bar{\Pi}_{i}\left(c_{n} a_{i}\right)}, \frac{1}{n \bar{\Pi}_{i}\left(c_{n} b_{i}\right)}\right] \rightarrow(-\infty, \infty)=: B_{5} \quad \text { for } i \in K_{5}, \\
& \left(\frac{1}{n \bar{\Pi}_{i}(0+)}, \frac{1}{n \bar{\Pi}_{i}\left(c_{n} b_{i}\right)}\right] \rightarrow\left(0, \frac{\operatorname{sgn}\left(b_{i}\right)}{p_{i}^{\operatorname{sgn}\left(b_{i}\right)}}\left|b_{i}\right|^{\alpha}\right]=: B_{6} \quad \text { for } i \in K_{6}, \\
& \left(\frac{1}{n \bar{\Pi}_{i}(0+)}, \frac{1}{n \bar{\Pi}_{i}\left(c_{n} b_{i}\right)}\right] \rightarrow(0, \infty)=: B_{7} \quad \text { for } i \in K_{7}, \\
& \left(\frac{1}{n \bar{\Pi}_{i}\left(c_{n} a_{i}\right)}, \frac{1}{n \bar{\Pi}_{i}(0+)}\right] \rightarrow\left(\frac{\operatorname{sgn}\left(a_{i}\right)}{p_{i}^{\operatorname{sgn}\left(a_{i}\right)}}\left|a_{i}\right|^{\alpha}, 0\right]=: B_{8} \quad \text { for } i \in K_{8}, \\
& \left(\frac{1}{n \bar{\Pi}_{i}\left(c_{n} a_{i}\right)}, \frac{1}{n \bar{\Pi}_{i}(0+)}\right] \rightarrow(-\infty, 0]=: B_{9} \quad \text { for } i \in K_{9} .
\end{aligned}
$$


Furthermore,

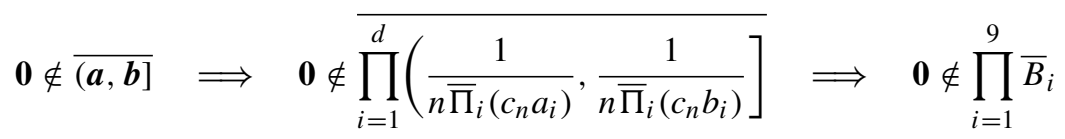

and

$$
\mu(\partial(\boldsymbol{a}, \boldsymbol{b}])=0 \Longrightarrow v\left(\partial \prod_{i=1}^{d}\left(\frac{1}{n \bar{\Pi}_{i}\left(c_{n} a_{i}\right)}, \frac{1}{\bar{\Pi}_{i}\left(c_{n} b_{i}\right)}\right]\right)=0 \quad \Longrightarrow \quad v\left(\partial \prod_{i=1}^{9} B_{i}\right)=0 .
$$

Since $n \Gamma(n \cdot) \stackrel{\mathrm{v}}{\rightarrow} v(\cdot)$ as $n \rightarrow \infty$ and $v$ has no atoms on the considered sets, by applying Propositions 4.2.1 and 4.2.2 of [8], it follows that expression (12) converges to $\mu$ and relation (10) holds.

The properties of $\mu$ can easily be seen: $\mu$ is an $\alpha$-homogeneous Radon measure on $\mathcal{B}(\mathbb{E})$ with $\mu\left(\overline{\mathbb{R}}^{d} \backslash \mathbb{R}^{d}\right)=0$ since $v$ is a 1 -homogeneous Lévy measure. Moreover, $\mu$ is a nonzero Radon measure because the one-dimensional margin $\mu_{1}$ is a nonzero measure.

The following result is a converse of Theorem 2 and extends Lemma 1.

Theorem 3. Let $\Pi$ be a d-dimensional Lévy measure with one-dimensional margins $\Pi_{i}$ for $i=$ $1, \ldots, d$, and let $\Gamma$ be the PLM constructed for $\Pi$ to satisfy (2). Suppose that $\Pi \in \operatorname{RV}\left(\alpha, c_{n}, \mu\right)$. Then the tail balance conditions (9) hold and $\Gamma \in \operatorname{RV}(1, n, v)$. For $\boldsymbol{a}, \boldsymbol{b} \in \mathbb{R}^{d}$ with $(\boldsymbol{a}, \boldsymbol{b}] \subset$ $\prod_{i=1}^{d} \mathscr{D}_{i}$ and $\mathscr{D}_{i}$ defined as in (5), the relation between $\mu$ and $v$ is given as

$$
v((\boldsymbol{a}, \boldsymbol{b}])=\mu((\hat{\boldsymbol{a}}, \hat{\boldsymbol{b}}]),
$$

where, for $i=1, \ldots, d$,

$$
\hat{a}_{i}:= \begin{cases}0 & \text { if } p_{i}^{\operatorname{sgn}\left(a_{i}\right)}=0 \\ \operatorname{sgn}\left(a_{i}\right)\left(p_{i}^{\operatorname{sgn}\left(a_{i}\right)} a_{i}\right)^{1 / \alpha} & \text { if } p_{i}^{\operatorname{sgn}\left(a_{i}\right)}>0 \\ \infty & \text { if } a_{i}>0, p_{i}^{\operatorname{sgn}\left(a_{i}\right)}=0 \\ -\infty & \text { if } a_{i}<0, p_{i}^{\operatorname{sgn}\left(a_{i}\right)}=0\end{cases}
$$

with the $\hat{b}_{i}$ defined similarly. For $\boldsymbol{a}, \boldsymbol{b} \in \mathbb{R}^{d} \backslash \prod_{i=1}^{d} \mathscr{D}_{i}$, we have $v((\boldsymbol{a}, \boldsymbol{b}])=\Gamma((\boldsymbol{a}, \boldsymbol{b}])$.

Proof. By Lemma 1, the tail balance conditions (9) hold with $p_{i}^{+}:=\mu_{i}(1)$ and $p_{i}^{-}:=$ $-\bar{\mu}_{i}(-1)$, and there exists at least one index $i_{*}$ such that $p_{i_{*}}^{+}+p_{i_{*}}^{-}>0$. Analogously to the proof of Theorem 2, we have to show that $n \Gamma(n(\boldsymbol{a}, \boldsymbol{b}]) \rightarrow v((\boldsymbol{a}, \boldsymbol{b}])$ as $n \rightarrow \infty$ for all $\boldsymbol{a}, \boldsymbol{b} \in \mathbb{R}^{d} \backslash\{\boldsymbol{0}\}, \mathbf{0} \notin \overline{(\boldsymbol{a}, \boldsymbol{b}]}$, and $v(\partial(\boldsymbol{a}, \boldsymbol{b}])=0$, where $v$ is a nonzero 1-homogeneous Radon measure with $v\left(\overline{\mathbb{R}} \backslash \mathbb{R}^{d}\right)=0$. Recall the definition of the sets $\mathscr{D}_{i}$ in (5). By relation (7), $\Gamma$ is 1-homogeneous on $\mathbb{R}^{d} \backslash \prod_{i=1}^{d} \mathscr{D}_{i}$ and so we define $v((\boldsymbol{a}, \boldsymbol{b}]):=\Gamma((\boldsymbol{a}, \boldsymbol{b}])$ for sets $(\boldsymbol{a}, \boldsymbol{b}] \subset\left(\mathbb{R}^{d} \backslash \prod_{i=1}^{d} \mathscr{D}_{i}\right)$. Furthermore, we define $v$ on $\mathscr{B}(\mathbb{E})$ by $v\left(\overline{\mathbb{R}}^{d} \backslash \mathbb{R}^{d}\right):=0$ and, for $(\boldsymbol{a}, \boldsymbol{b}] \subset \prod_{i=1}^{d} \mathscr{D}_{i}$, we set, defining $\tilde{x}_{i}$ as in (11),

$$
v((\boldsymbol{a}, \boldsymbol{b}]):=\mu\left(\left\{\left(x_{1}, \ldots, x_{d}\right) \in \mathbb{R}^{d} \backslash\{\mathbf{0}\}: \tilde{x}_{i} \in\left(a_{i}, b_{i}\right] \text { for } i=1, \ldots, d\right\}\right) .
$$

Here $v$ is a 1-homogeneous Radon measure since $\mu$ is an $\alpha$-homogeneous Lévy measure and $\Gamma$ is 1-homogeneous on $\mathbb{R}^{d} \backslash \prod_{i=1}^{d} \mathscr{D}_{i}$. Moreover, $v$ is a nonzero measure because $\mu_{i_{*}}$ is nonzero 
and $p_{i_{*}}^{+}+p_{i_{*}}^{-}>0$. Suppose that $\boldsymbol{a}, \boldsymbol{b} \in \mathbb{R}^{d} \backslash\{\boldsymbol{0}\}$ with $\mathbf{0} \notin \overline{(\boldsymbol{a}, \boldsymbol{b}]}$ and $v(\partial(\boldsymbol{a}, \boldsymbol{b}])=0$. With relation (6) we obtain, for $(\boldsymbol{a}, \boldsymbol{b}] \subset \prod_{i=1}^{d} \mathscr{D}_{i}$,

$$
\begin{aligned}
& n \Gamma(n(\boldsymbol{a}, \boldsymbol{b}]) \\
& =\left.n \Pi \otimes \lambda\right|_{[0,1]^{d}}\left(\left\{\left(c_{n} x_{1}, \ldots, c_{n} x_{d}, y_{1}, \ldots, y_{d}\right) \in\left(\mathbb{R}^{d} \backslash\{\mathbf{0}\}\right) \times[0,1]^{d}:\right.\right. \\
& \left.\left.\frac{1}{n \dot{\bar{\Pi}}_{i}\left(c_{n} x_{i}\right)+n y_{i} \Delta \bar{\Pi}_{i}\left(c_{n} x_{i}\right)} \in\left(a_{i}, b_{i}\right] \text { for } i=1, \ldots, d\right\}\right) .
\end{aligned}
$$

With $\lim _{n \rightarrow \infty} n \Delta \bar{\Pi}_{i}\left(c_{n} x_{i}\right)=0$ we have

$$
\lim _{n \rightarrow \infty} \frac{1}{n \dot{\bar{\Pi}}_{i}\left(c_{n} x_{i}\right)+y_{i} \Delta \bar{\Pi}_{i}\left(c_{n} x_{i}\right)}= \begin{cases}0 & \text { if } x_{i}=0, \\ \operatorname{sgn}\left(x_{i}\right)\left(p_{i}^{\operatorname{sgn}\left(x_{i}\right)}\right)^{-1}\left|x_{i}\right|^{\alpha} & \text { if } x_{i} \neq 0, p_{i}^{\operatorname{sgn}\left(x_{i}\right)}>0 \\ \infty & \text { if } x_{i}>0, p_{i}^{\operatorname{sgn}\left(x_{i}\right)}=0 \\ -\infty & \text { if } x_{i}<0, p_{i}^{\operatorname{sgn}\left(x_{i}\right)}=0\end{cases}
$$

We see that $v(\partial(\boldsymbol{a}, \boldsymbol{b}])=0$ holds if and only if $\mu(\partial(\hat{\boldsymbol{a}}, \hat{\boldsymbol{b}}])=0$ and $\mathbf{0} \notin(\boldsymbol{a}, \boldsymbol{b}]$ implies that $\mathbf{0} \notin(\hat{\boldsymbol{a}}, \hat{\boldsymbol{b}}]$. So, with Propositions 4.2.1 and 4.2.3 of [8], it follows, for (14) as $n \rightarrow \infty$, that

$$
\begin{array}{r}
\lim _{n \rightarrow \infty} n \Gamma(n(\boldsymbol{a}, \boldsymbol{b}])=\left.\mu \otimes \lambda\right|_{[0,1]^{d}}\left(\left\{\left(x_{1}, \ldots, x_{d}, y_{1}, \ldots, y_{d}\right) \in\left(\mathbb{R}^{d} \backslash\{\boldsymbol{0}\}\right) \times[0,1]^{d}:\right.\right. \\
\left.\left.\tilde{x}_{i} \in\left(a_{i}, b_{i}\right] \text { for } i=1, \ldots, d\right\}\right)=v((\boldsymbol{a}, \boldsymbol{b}]),
\end{array}
$$

and (13) follows.

We conclude this section with an account of extreme (jump) dependence between components of a Lévy process. In analogy to the definition of the tail dependence coefficient for distribution functions (see, e.g. [18]) we define extreme dependence measures for different situations. The definition is usually restricted to dimension $d=2$.

Definition 5. (Tail dependence coefficients.) Let $\Pi$ be a Lévy measure in $\mathbb{R}^{d}$ with equal marginals. We define its upper and lower tail dependence coefficients as

$$
\Lambda_{\mathrm{U}}:=\lim _{t \rightarrow \infty} \frac{\bar{\Pi}(t, \ldots, t)}{\bar{\Pi}_{1}(t)} \quad \text { and } \quad \Lambda_{\mathrm{L}}:=\lim _{t \rightarrow-\infty} \frac{\bar{\Pi}(t, \ldots, t)}{\bar{\Pi}_{1}(t)},
$$

respectively, provided that these limits exist. If $\Lambda_{\mathrm{U}}>0$, we call $\Pi$ upper tail dependent, and if $\Lambda_{\mathrm{L}}>0$ the PLM $\Pi$ is called lower tail dependent.

Recall that, for multivariate distributions, the tail dependence coefficient is a copula parameter. The following result shows that the analogous result holds for Lévy measures: the tail dependence coefficients are PLC parameters. It is a simple consequence of the definitions.

Proposition 3. Assume that $\Pi$ is a Lévy measure in $\mathbb{R}^{d}$ with equal marginals and with PLM $\Gamma$. Then

$$
\Lambda_{\mathrm{U}}=\lim _{t \rightarrow \infty} t \bar{\Gamma}(t, \ldots, t) \quad \text { and } \quad \Lambda_{\mathrm{L}}=\lim _{t \rightarrow-\infty}|t \bar{\Gamma}(t, \ldots, t)| .
$$

Moreover, both limits exist.

The picture changes when we deal with nonequal marginals as in Theorem 2. 
Proposition 4. (i) Assume that $\Pi \in \mathrm{RV}\left(\alpha, c_{n}, \mu\right)$, one of the marginals, say, $\Pi_{1} \in \mathrm{RV}\left(\alpha, c_{n}, \mu\right)$, and the tail balance condition (9) holds for all marginals. Then the PLM $\Gamma \in \operatorname{RV}(1, n, v)$ and

$$
\begin{aligned}
& \lim _{t \rightarrow \infty} \frac{\bar{\Pi}(t, \ldots, t)}{\bar{\Pi}_{1}(t)}=\frac{\bar{\mu}(1, \ldots, 1)}{\bar{\mu}_{1}(1)}=\bar{v}\left(1, \frac{p_{2}^{+}}{p_{d}^{+}}, \ldots, \frac{p_{1}^{+}}{p_{d}^{+}}\right), \\
& \lim _{t \rightarrow-\infty} \frac{\bar{\Pi}(t, \ldots, t)}{\bar{\Pi}_{1}(t)}=\frac{\bar{\mu}(-1, \ldots,-1)}{\bar{\mu}_{1}(-1)}=\bar{v}\left(-1,-\frac{p_{1}^{-}}{p_{d}^{-}}, \ldots,-\frac{p_{1}^{-}}{p_{d}^{-}}\right),
\end{aligned}
$$

and both limits exist. If $p_{i}^{+}=0$ or $p_{i}^{-}=0$ for some $i \in\{2, \ldots, d\}$, then the corresponding component is interpreted as $\infty$ and it does not contribute to the limit.

(ii) For a Lévy measure $\Pi$ with equal marginals, the limits in (15) and (16) are $\Lambda_{\mathrm{U}}$ and $\Lambda_{\mathrm{L}}$, respectively.

Note also that the representations on the right-hand sides of (15) and (16) show that the weights of the $d$ marginals play an important role, as well as the tail integral of the limit measure $v$ of the PLM $\Gamma$.

By Theorem 5.1 of [17], $X_{1} \in \operatorname{RV}\left(\alpha, c_{n}, \mu\right)$ if and only if $\Pi \in \operatorname{RV}\left(\alpha, c_{n}, \mu\right)$; hence, the following holds.

Corollary 1. (i) Let $\left(\boldsymbol{X}_{t}\right)_{t \geq 0}$ be the Lévy process with Lévy measure П. Assume that $\boldsymbol{X}_{\mathbf{1}}=$ $\left(X_{11}, \ldots, X_{1 d}\right)$ has equally distributed marginals. Recall that the upper and lower tail dependence coefficients of $\boldsymbol{X}_{\mathbf{1}}$ are defined as

$$
\lim _{x \rightarrow \infty} \frac{\mathrm{P}\left(X_{11}>x, \ldots, X_{1 d}>x\right)}{\mathrm{P}\left(X_{11}>x\right)} \text { and } \lim _{x \rightarrow-\infty} \frac{\mathrm{P}\left(X_{11} \leq x, \ldots, X_{1 d} \leq x\right)}{\mathrm{P}\left(X_{11} \leq x\right)} \text {, }
$$

respectively, provided that the limit exists. Assume that $\boldsymbol{X}$ is regularly varying with $\boldsymbol{X}_{\mathbf{1}} \in$ $\mathrm{RV}\left(\alpha, c_{n}, \mu\right)$. Then the limits in (17) both exist and are $\Lambda_{\mathrm{U}}$ and $\Lambda_{\mathrm{L}}$, respectively.

(ii) The results of Proposition 4(ii) hold for the distribution tails in the spirit of (17).

\section{Examples}

To simplify notation, we consider only the case $d=2$. Moreover, we assume that we are in the framework of Theorem 2 with $\Pi_{1} \in \mathrm{RV}\left(\alpha, c_{n}, \mu_{1}\right), p_{1}^{+}, p_{1}^{-}>0$, and $p_{2}^{+}, p_{2}^{-} \geq 0$, i.e. $\mu_{i}(x)=\operatorname{sgn}(x) p_{i}^{\operatorname{sgn}(x)}|x|^{-\alpha}$ for $x \neq 0$.

Example 6. (Example 1 continued: independence PLM.) Since $\Gamma_{\perp}$ is homogeneous of degree 1 , by Theorem 2 we obtain $\Pi \in \operatorname{RV}\left(\alpha, c_{n}, \mu\right)$ and, with

$$
\Gamma_{\perp}\left(\mathrm{d} x_{1}, \mathrm{~d} x_{2}\right)=\delta_{0}\left(\mathrm{~d} x_{1}\right)\left|x_{2}\right|^{-2} \mathrm{~d} x_{2}+\delta_{0}\left(\mathrm{~d} x_{2}\right)\left|x_{1}\right|^{-2} \mathrm{~d} x_{1}, \quad\left(x_{1}, x_{2}\right) \in \mathbb{R}^{2} \backslash\{\mathbf{0}\},
$$

the limit measure $\mu$ is supported on the axes. Hence, $\mu$ is given by

$$
\mu\left(\mathrm{d} x_{1}, \mathrm{~d} x_{2}\right)=\delta_{0}\left(\mathrm{~d} x_{1}\right) \frac{\alpha p_{2}^{\operatorname{sgn}\left(x_{2}\right)}}{\left|x_{2}\right|^{\alpha+1}} \mathrm{~d} x_{2}+\delta_{0}\left(\mathrm{~d} x_{2}\right) \frac{\alpha p_{1}^{\operatorname{sgn}\left(x_{1}\right)}}{\left|x_{1}\right|^{\alpha+1}} \mathrm{~d} x_{1}, \quad\left(x_{1}, x_{2}\right) \in \mathbb{R}^{2} \backslash\{\boldsymbol{0}\} .
$$

Then the upper and lower tail dependence coefficients, $\Lambda_{\mathrm{U}}$ and $\Lambda_{\mathrm{L}}$, equal 0 . 
Example 7. (Example 2 continued: complete positive dependence PLM.) $\Gamma_{\|}$is homogeneous of degree 1 and with (10) we obtain

$\mu\left(\mathcal{I}\left(x_{1}\right) \times \mathcal{I}\left(x_{2}\right)\right)=\left(p_{1}^{\operatorname{sgn}\left(x_{1}\right)}\left|x_{1}\right|^{-\alpha} \wedge p_{2}^{\operatorname{sgn}\left(x_{2}\right)}\left|x_{2}\right|^{-\alpha}\right) 1_{K}\left(\left(x_{1}, x_{2}\right)\right), \quad\left(x_{1}, x_{2}\right) \in(\mathbb{R} \backslash\{0\})^{2}$.

For $x_{1} \in \mathbb{R} \backslash\{0\}$, we have

$$
\begin{aligned}
\mu\left(\mathcal{I}\left(x_{1}\right) \times\{0\}\right) & =\mu_{1}\left(\mathcal{I}\left(x_{1}\right)\right)-\lim _{x_{2} \uparrow 0} \mu\left(\mathcal{I}\left(x_{1}\right) \times \mathcal{I}\left(x_{2}\right)\right)-\lim _{x_{2} \downarrow 0} \mu\left(\mathcal{I}\left(x_{1}\right) \times \mathcal{I}\left(x_{2}\right)\right) \\
& = \begin{cases}p_{1}^{\operatorname{sgn}\left(x_{1}\right)}\left|x_{1}\right|^{-\alpha} & \text { if } p_{2}^{\operatorname{sgn}\left(x_{1}\right)}=0, \\
0 & \text { if } p_{2}^{\operatorname{sgn}\left(x_{1}\right)}>0 .\end{cases}
\end{aligned}
$$

Analogously, for $x_{2} \in \mathbb{R} \backslash\{0\}$ with $p_{1}^{+}>0$ and $p_{1}^{-}>0$, we obtain $\mu\left(\{0\} \times \mathcal{I}\left(x_{2}\right)\right)=0$. Since $\Gamma_{\|}$ is supported by $\left\{\left(x_{1}, x_{2}\right) \in(\mathbb{R} \backslash\{0\})^{2}: x_{1}=x_{2}\right\}, \mu$ is supported by $\left\{\left(x_{1}, x_{2}\right) \in(\mathbb{R} \backslash\{0\})^{2}: x_{2}=\right.$ $\left.\left(p_{2}^{\operatorname{sgn}\left(x_{2}\right)} / p_{1}^{\operatorname{sgn}\left(x_{1}\right)}\right)^{1 / \alpha} x_{1}\right\}$. Finally, the limit measure $\mu$ results in

$$
\mu\left(\mathrm{d} x_{1}, \mathrm{~d} x_{2}\right)=p_{1}^{\operatorname{sgn}\left(x_{1}\right)} \frac{\alpha}{\left|x_{1}\right|^{\alpha+1}} 1_{\left\{x_{2}=\left(p_{2}^{\operatorname{sgn}\left(x_{2}\right)} / p_{1}^{\operatorname{sgn}\left(x_{1}\right)}\right)^{1 / \alpha} x_{1}\right\}} \mathrm{d} x_{1}, \quad x_{1} \in \mathbb{R} \backslash\{0\} .
$$

Then the limits in (15) and (16) are given as

$$
\lim _{t \rightarrow \infty} \frac{\bar{\Pi}(t, t)}{\bar{\Pi}_{1}(t)}=\frac{p_{1}^{+} \wedge p_{2}^{+}}{p_{1}^{+}} \text {and } \lim _{t \rightarrow-\infty} \frac{\bar{\Pi}(t, t)}{\bar{\Pi}_{1}(t)}=-\frac{p_{1}^{-} \wedge p_{2}^{-}}{p_{1}^{-}} .
$$

Example 8. (Example 4 continued: Clayton PLM.) The Clayton PLM $\Gamma_{\eta, \theta}$ is homogeneous of degree 1 and we have $\Gamma_{\eta, \theta}\left(\mathbb{R}^{2} \backslash(\mathbb{R} \backslash\{0\})^{2}\right)=0$. For $x_{1} \in \mathbb{R} \backslash\{0\}$, we obtain

$$
\begin{aligned}
& \mu\left(\mathcal{I}\left(x_{1}\right) \times\{0\}\right)=\lim _{\varepsilon \uparrow 0} \mu\left(\mathcal{I}\left(x_{1}\right) \times(\varepsilon, 0]\right)
\end{aligned}
$$

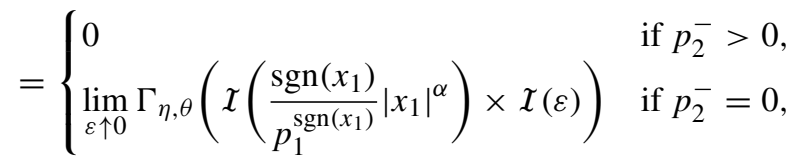

$$
\begin{aligned}
& = \begin{cases}0 & \text { if } p_{2}^{-}>0, \\
p_{1}^{\operatorname{sgn}\left(x_{1}\right)}\left|x_{1}\right|^{-\alpha}\left(\eta 1_{\left\{x_{1}<0\right\}}+(1-\eta) 1_{\left\{x_{1}>0\right\}}\right) & \text { if } p_{2}^{-}=0,\end{cases}
\end{aligned}
$$

and, for $x_{2} \in \mathbb{R} \backslash\{0\}$, we obtain

$$
\begin{aligned}
\mu\left(\{0\} \times \mathcal{I}\left(x_{2}\right)\right) & =\lim _{\varepsilon \uparrow 0} \mu\left((\varepsilon, 0] \times \mathcal{I}\left(x_{2}\right)\right) \\
& =\lim _{\varepsilon \uparrow 0} \Gamma\left(\left(\frac{-1}{p_{1}^{-}}|\varepsilon|^{\alpha}, 0\right] \times \mathcal{I}\left(\tilde{x}_{2}\right)\right) \\
& =\Gamma_{\eta, \theta}\left(\{0\} \times \mathcal{L}\left(\tilde{x}_{2}\right)\right) \\
& =0 .
\end{aligned}
$$

Let $x_{1}, x_{2} \in \mathbb{R} \backslash\{0\}$. If $p_{2}^{\operatorname{sgn}\left(x_{2}\right)}>0$ then

$$
\begin{aligned}
\mu\left(\mathcal{I}\left(x_{1}\right) \times \mathcal{I}\left(x_{2}\right)\right)= & \left(\left(p_{1}^{\operatorname{sgn}\left(x_{1}\right)}\right)^{-\theta}\left|x_{1}\right|^{\alpha \theta}+\left(p_{2}^{\operatorname{sgn}\left(x_{2}\right)}\right)^{-\theta}\left|x_{2}\right|^{\alpha \theta}\right)^{-1 / \theta} \\
& \times\left(\eta 1_{\left\{x_{1} x_{2}>0\right\}}+(1-\eta) 1_{\left\{x_{1} x_{2}<0\right\}}\right) .
\end{aligned}
$$


If $p_{i}^{\operatorname{sgn}\left(x_{2}\right)}=0$ then

$$
\mu\left(\mathcal{I}\left(x_{1}\right) \times \mathcal{L}\left(x_{2}\right)\right)=\Gamma_{\eta, \theta}\left(\mathcal{I}\left(\frac{\operatorname{sgn}\left(x_{1}\right)}{p_{1}^{\operatorname{sgn}\left(x_{1}\right)}}\left|x_{1}\right|^{\alpha}\right) \times \varnothing\right)=0 .
$$

Moreover, the limits in (15) and (16) are given by

$$
\lim _{t \rightarrow \infty} \frac{\bar{\Pi}(t, t)}{\bar{\Pi}_{1}(t)}=\frac{\bar{\mu}(1,1)}{\bar{\mu}(1)}= \begin{cases}\frac{\eta\left(\left(p_{1}^{+}\right)^{-\theta}+\left(p_{2}^{+}\right)^{-\theta}\right)^{-1 / \theta}}{p_{1}^{+}} & \text {if } p_{2}^{+}>0 \\ 0 & \text { if } p_{2}^{+}=0\end{cases}
$$

and

$$
\lim _{t \rightarrow-\infty} \frac{\bar{\Pi}(t, t)}{\bar{\Pi}_{1}(t)}=\frac{\bar{\mu}(-1,-1)}{\bar{\mu}(-1)}= \begin{cases}\frac{-\eta\left(\left(p_{1}^{-}\right)^{-\theta}+\left(p_{2}^{-}\right)^{-\theta}\right)^{-1 / \theta}}{p_{1}^{-}} & \text {if } p_{2}^{-}>0, \\ 0 & \text { if } p_{2}^{-}=0,\end{cases}
$$

and $\Lambda_{\mathrm{U}}=\Lambda_{\mathrm{L}}=\eta 2^{-1 / \theta}$. So, if $\eta>0$ and $p_{2}^{+}>0\left(p_{2}^{-}>0\right)$, then we always have upper (lower) tail dependence.

Example 9. (Example 5 continued: nonhomogeneous $P L M$.) $\Gamma_{\eta, \zeta}$ is concentrated on $(\mathbb{R} \backslash\{0\})^{2}$ and we obtain

$$
\lim _{n \rightarrow \infty} n \Gamma_{\eta, \zeta}\left(n\left(\mathcal{I}\left(x_{1}\right) \times \mathcal{L}\left(x_{2}\right)\right)\right)=\frac{1}{\left|x_{1}\right|+\left|x_{2}\right|+n\left|x_{1} x_{2}\right|}=0, \quad x_{1}, x_{2} \in \mathbb{R} \backslash\{0\} .
$$

Therefore, $\Gamma_{\eta, \zeta}$ is not only nonhomogeneous, but also not regularly varying. Consequently, by Theorem 2, $\Pi$ defined by (2) is not multivariate regularly varying. Moreover, $\Lambda_{\mathrm{U}}=\Lambda_{\mathrm{L}}=0$, so there is no tail dependence in this model.

It was shown in [9] that, for $\eta=1$, the Lévy measure of the sum of the two components of the bivariate Lévy process can, for certain marginal models, be calculated explicitly. This is also true for the PLM. Surprisingly, it turns out that the Lévy measure of the sum

$$
\Gamma_{+}(\cdot):=\Gamma_{1, \zeta}\left(\left\{\left(x_{1}, x_{2}\right) \in \mathbb{R}^{2} \backslash\{\mathbf{0}\}: x_{1}+x_{2} \in \cdot\right\}\right)
$$

is univariate regularly varying. Note that this does not contradict [3, Theorem 1.1], where it was proved that a vector $\boldsymbol{X}$ is regularly varying, if and only if every linear combination is regularly varying. More precisely, for $z>0$,

$$
\bar{\Gamma}_{+}(z)=\frac{6+2 z \zeta}{z(4+z \zeta)}+\frac{4+2 z \zeta}{z(4+z \zeta) \sqrt{z \zeta(4+z \zeta)}} \ln \left(\left|\frac{z \zeta+\sqrt{z \zeta(4+z \zeta)}}{z \zeta-\sqrt{z \zeta(4+z \zeta)}}\right|\right) .
$$

From this, it is easy to see that $\bar{\Gamma}_{+}(z) \sim 2 z^{-1}=\bar{\Gamma}_{1}(z)+\bar{\Gamma}_{2}(z)$, which exhibits the same behavior as the independence model.

\section{Graphical representation of the dependence structure of Lévy processes}

For a stable random vector, the spectral measure characterizes the dependence between the marginals (see [25, Definition 2.3.2]), which remains true for a regularly varying random vector in the limit; see Definition 1. Consequently, the spectral density has been presented graphically for stable and regularly varying distributions and processes, at least in two dimensions.

The PLC provides a new possibility to visualize the dependence structure between the jump parts of the marginal Lévy processes. As a graphical tool, it can also be applied to nonregularly varying PLMs, where no spectral measure exists. Whereas an empirical version of the spectral 
density estimates a density, immediately by definition, an empirical version of the PLC estimates a tail integral. This means that it always exists, also if the spectral measure has no density, or if the PLC is not homogeneous of any order. Moreover, the dependence of joint extremes is estimated by the tail dependence coefficients, which is based on the PLC and the tail integral as indicated in Definition 5 and Corollary 4.

In the first subsection below we present both graphical representations for bivariate homogeneous PLMs from the previous examples, the spectral density, and the PLC. In the second subsection we visualize the dependence structure of the nonregularly varying PLM given in Example 5 only by its PLC, since there exists no spectral density.

\subsection{Homogeneous Pareto Lévy measures}

Recall from Theorem 14.3 of [26] that the 1-homogeneous PLM $\Gamma$ as a 1-stable Lévy measure has, for all $B \in \mathcal{B}\left(\mathbb{R}^{d}\right)$, the representation $\Gamma(B)=\int_{\mathbb{S}} \int_{0}^{\infty} 1_{B}(r \phi) r^{-2} \mathrm{~d} r \tilde{\mu}_{\mathbb{S}}(\mathrm{d} \phi)$, where $\mathbb{S}$ denotes the unit sphere in $\mathbb{R}^{d}$. Since $\tilde{\mu}_{\mathbb{S}}$ is a finite measure, it can be normalized to a probability measure, which means in our situation that

$$
\mu_{\mathbb{S}}(\cdot)=\frac{\tilde{\mu}_{\mathbb{S}}(\cdot)}{\Gamma\left(\left\{\boldsymbol{x} \in \mathbb{R}^{2}:|\boldsymbol{x}|>1\right\}\right)}=\frac{\Gamma\left(\left\{\boldsymbol{x} \in \mathbb{R}^{2}:|x|>1, \boldsymbol{x} /|\boldsymbol{x}| \in \cdot\right\}\right)}{\Gamma\left(\left\{\boldsymbol{x} \in \mathbb{R}^{2}:|\boldsymbol{x}|>1\right\}\right)} .
$$

This representation shows that $\mu_{\mathbb{S}}$ measures the dependence between joint extremes, and that it depends on the chosen norm $|\cdot|$.

Using polar coordinates $r=|\boldsymbol{x}|$ and $\phi=\boldsymbol{x} /|\boldsymbol{x}| \in \mathbb{S}$, the PLM $\Gamma$ has, for the set $A:=$ $\left\{(r, \phi): 0 \leq r_{1} \leq r \leq r_{2} \leq \infty, 0 \leq \rho_{1} \leq \phi \leq \rho_{2}<2 \pi\right\}$, the representation

$$
\Gamma(A)=\int_{\rho_{1}}^{\rho_{2}} \int_{r_{1}}^{r_{2}} r^{-2} \mathrm{~d} r \tilde{\mu}_{\mathbb{S}}(\mathrm{d} \phi) .
$$

Note that all sets $A$ of this type form a semialgebra of subsets of $\mathbb{R}^{2} \backslash\{\boldsymbol{0}\}$ and, hence, generate the Borel $\sigma$-algebra $\mathscr{B}\left(\mathbb{R}^{2} \backslash\{\mathbf{0}\}\right)$. Defining the transformation $T:[0, \infty) \times[0,2 \pi) \rightarrow \mathbb{R}^{2}$ by $T(r, \phi)=r(\cos \phi, \sin \phi), \Gamma$ has a density given in polar coordinates as

$$
\Gamma \circ T(\mathrm{~d} r, \mathrm{~d} \phi)=\tilde{\mu}_{\mathbb{S}}(\mathrm{d} \phi) r^{-2} \mathrm{~d} r .
$$

From this we see firstly the well-known fact that the spectral density as the normalized angular measure completely determines the dependence in 1-stable models (as in all homogeneous models of any order). We also note that the homogeneity on the whole of $\mathbb{R}^{d}$ plays an important role for the multiplicative structure in (20): for a general PLM, this no longer holds.

Using the notation introduced above, we can relate the spectral measure with the PLM as follows. Since the arcs are for any norm given by

$$
S_{\rho_{1}, \rho_{2}}:=\left\{\frac{(\cos \phi, \sin \phi)}{|(\cos \phi, \sin \phi)|}: \rho_{1}<\phi \leq \rho_{2}\right\}
$$

we find, from (18) by integrating out $r$ over $(1, \infty)$ in (19), that

$$
\mu_{\mathbb{S}}\left(\left[\rho_{1}, \rho_{2}\right]\right)=\frac{\int_{\rho_{1}}^{\rho_{2}} \tilde{\mu}_{\mathbb{S}}(\mathrm{d} \phi)}{\int_{0}^{2 \pi} \tilde{\mu}_{\mathbb{S}}(\mathrm{d} \phi)}=\frac{\Gamma\left(S_{\rho_{1}, \rho_{2}}\right)}{\int_{0}^{2 \pi} \tilde{\mu}_{\mathbb{S}}(\mathrm{d} \phi)} .
$$

We present the spectral measures by plotting the density $\mu_{\mathbb{S}}(\mathrm{d} \phi) / \mathrm{d} \phi$ on $[0,2 \pi)$. Here we take an idea from [2] and visualize $\mu_{\mathbb{S}}$ as a graph such that the area included between two angles $\left(\rho_{1}\right.$ and $\rho_{2}$, say) and a solid curve $\left(s(\rho)\right.$ for $\left.\rho \in\left[\rho_{1}, \rho_{2}\right]\right)$ represents the spectral measure $\mu_{\mathbb{S}}\left(\left[\rho_{1}, \rho_{2}\right]\right)$. The uniform distribution corresponds then to the unit circle. We call these graphs Basrak graphs. 
Whereas polar coordinates are the natural coordinates for the spectral measure, the natural coordinates for the PLC $\bar{\Gamma}$ are the Cartesian coordinates. The natural generator of the Borel $\sigma$-algebra $\mathcal{B}\left(\mathbb{R}^{2} \backslash\{\boldsymbol{0}\}\right)$ are here the sets, which have been used to define the tail integral. It is, however, obvious from the definition of the spectral measure in (18) that there is no simple relation between the spectral measure and the PLM on these sets. For specific PLCs, it is possible (as shown below) to obtain the form of the corresponding spectral measure, provided that the homogeneity property holds.

On the other hand, for nonhomogeneous PLCs, there exists no spectral measure. More precisely, the factorization of the PLM into a radial part and an angular part is no longer possible: the angular part still depends on $r$; cf. Example 13. However, the PLC $\bar{\Gamma}$ exists and can also be visualized. Consequently, we also suggest a graphic representation for the PLC. This is done as follows. For a given point $\left(x_{1}, x_{2}\right)$ not on any axes, we plot $\left|\bar{\Gamma}\left(x_{1}, x_{2}\right)\right|=\Gamma\left(\mathcal{I}\left(x_{1}\right) \times \mathcal{I}\left(x_{2}\right)\right)$ as the $L_{2}$-distance of a point to the origin, where we can also use any other distance.

Example 10. (Examples 1 and 6 continued: independence PLM.) Since $\Gamma_{\perp}$ has mass only on the axes, its spectral measure is, for $\phi \in[0,2 \pi)$, given by

$$
\mu_{\mathbb{S}}(\mathrm{d} \phi)=\frac{1}{4} \delta_{0}(\mathrm{~d} \phi)+\frac{1}{4} \delta_{\pi / 2}(\mathrm{~d} \phi)+\frac{1}{4} \delta_{\pi}(\mathrm{d} \phi)+\frac{1}{4} \delta_{3 \pi / 2}(\mathrm{~d} \phi) .
$$

Figure 1 shows the spectral density $\mu_{\mathbb{S}}(\mathrm{d} \phi) / \mathrm{d} \phi$. The PLC $\bar{\Gamma}_{\perp}$ is equal to 0 ; see Figure 2 .

Example 11. (Examples 2 and 7 continued: complete positive dependence PLM.) $\Gamma_{\|}$has mass only on $\left\{\boldsymbol{x} \in \mathbb{R}^{2} \backslash\{\boldsymbol{0}\}: x_{1}=x_{2}\right\}$ and its spectral measure is, for $\phi \in[0,2 \pi)$, given by $\mu_{\mathbb{S}}(\mathrm{d} \phi)=\frac{1}{2} \delta_{\pi / 4}(\mathrm{~d} \phi)+\frac{1}{2} \delta_{5 \pi / 4}(\mathrm{~d} \phi)$. The right diagram of Figure 1 shows the spectral density $\mu_{\mathbb{S}}(\mathrm{d} \phi) / \mathrm{d} \phi$ for angles in $[0,2 \pi)$ and as Basrak graph. The PLC $\bar{\Gamma}_{\|}$is given as $\bar{\Gamma}_{\|}\left(x_{1}, x_{2}\right)=1_{K}\left(\left(x_{1}, x_{2}\right)\right) \operatorname{sgn}\left(x_{1}\right) \operatorname{sgn}\left(x_{2}\right) /\left(\left|x_{1}\right| \vee\left|x_{2}\right|\right)$, and is visualized in Figure 2.

Example 12. (Examples 4 and 8 continued: Clayton PLM.) From (8) we find that

$$
\begin{aligned}
\Gamma_{\eta, \theta}\left(\mathrm{d} x_{1}, \mathrm{~d} x_{2}\right)= & (1+\theta)\left(\left|x_{1}\right|^{\theta}+\left|x_{2}\right|^{\theta}\right)^{-1 / \theta-2}\left|x_{1}\right|^{\theta-1}\left|x_{2}\right|^{\theta-1} \\
& \times\left(\eta 1_{\left\{x_{1} x_{2}>0\right\}}+(1-\eta) 1_{\left\{x_{1} x_{2}<0\right\}}\right) \mathrm{d} x_{1} \mathrm{~d} x_{2} .
\end{aligned}
$$
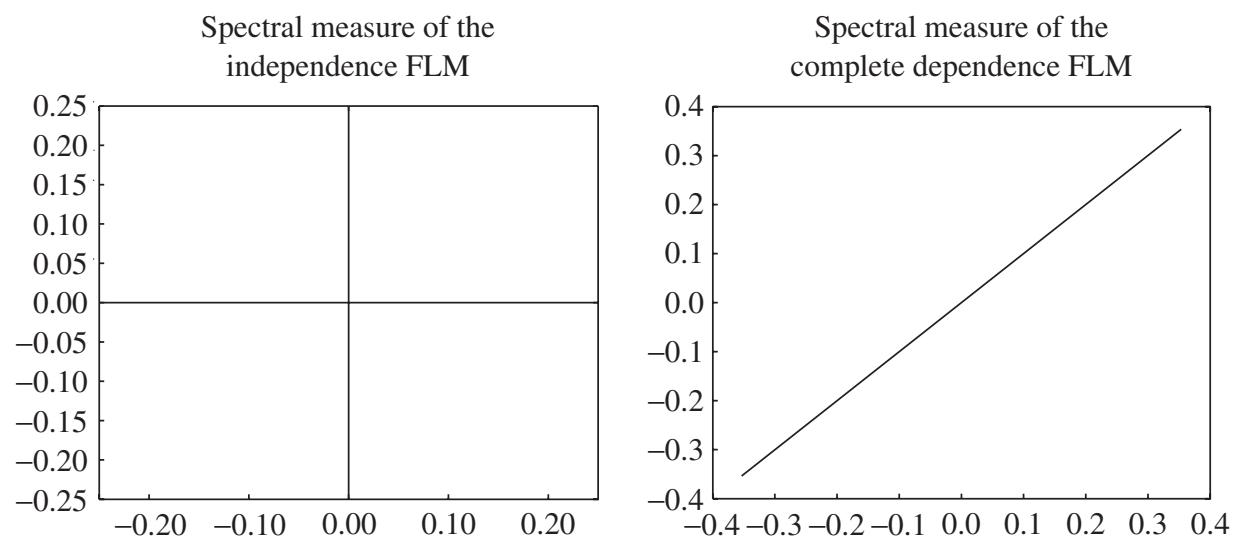

FIGURE 1: The left diagram shows the Basrak graph of the spectral measure $\mu_{\mathbb{S}}$ of the independence PLM in $[0,2 \pi)$ with uniform weights 0.25 on $0, \frac{1}{2} \pi, \pi, \frac{3}{2} \pi$. The right diagram shows the Basrak plot of the spectral measure $\mu_{\mathbb{S}}$ of the complete positive dependence PLM. The length of the rays represents the probability mass on the corresponding angles. 
PLC of independence and complete dependence

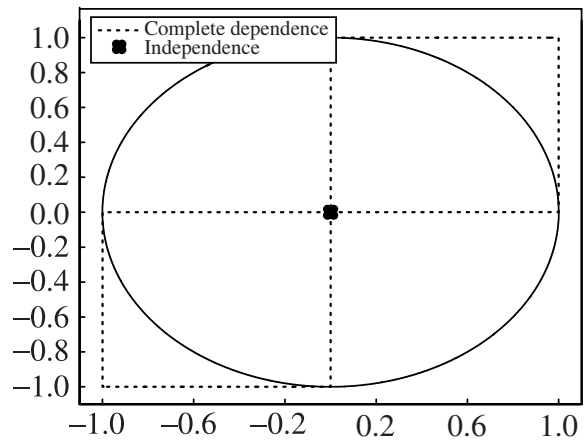

Clayton PLC on $S_{2}, \theta=0.5$

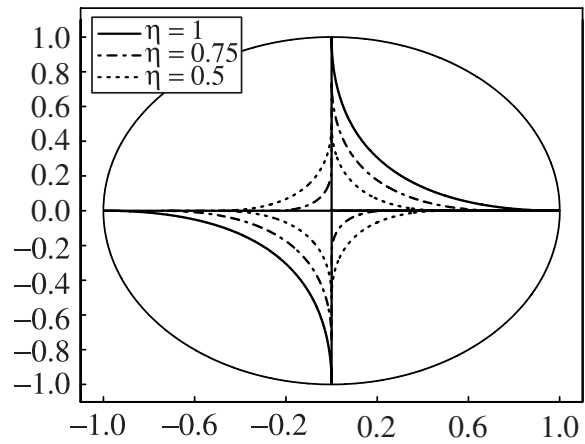

Clayton PLC on $S_{2}, \theta=2$

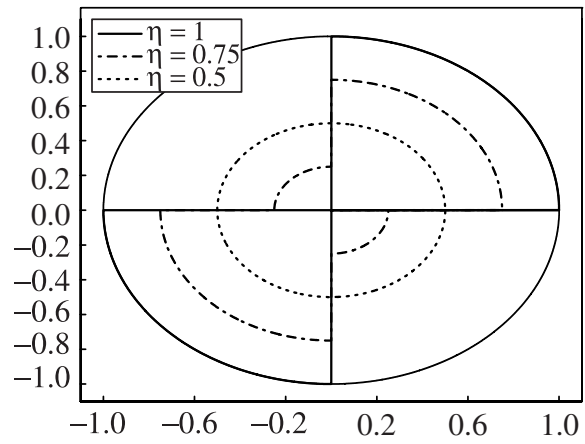

Clayton PLC on $S_{2}, \eta=1$

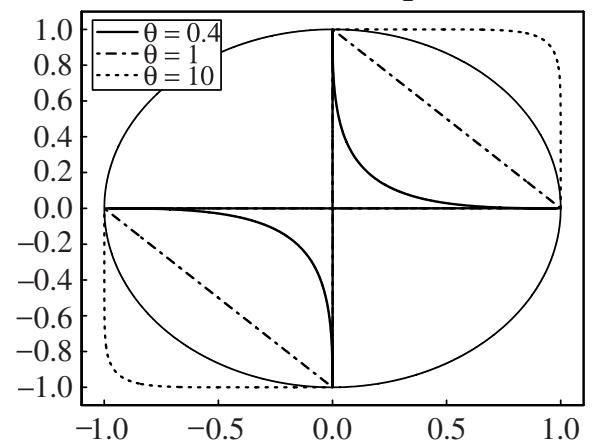

Clayton PLC on $S_{2}, \theta=1$

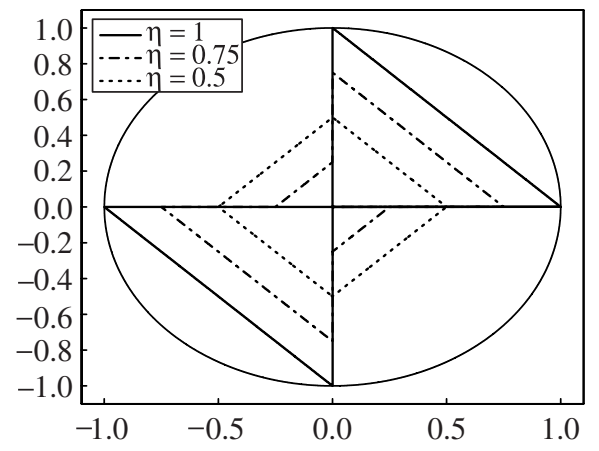

Clayton PLC on $S_{2}, \theta=5$

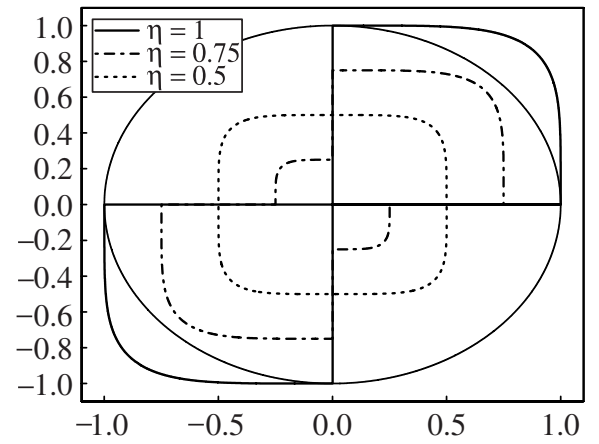

Figure 2: The first row of diagrams shows the PLC $\bar{\Gamma}_{\perp}, \bar{\Gamma}_{\|}($left $)$, and $\bar{\Gamma}_{\eta, \theta}$ for $\eta=1$ and different values $\theta>0($ right $)$. The other diagrams show $\bar{\Gamma}_{\eta, \theta}$ for different values of $\theta$ and $\eta \in[0,1]$.

By a transformation to polar coordinates, $\Gamma_{\eta, \theta}$ has representation (20) with density

$$
\begin{aligned}
\frac{\tilde{\mu}_{\mathbb{S}}(\mathrm{d} \phi)}{\mathrm{d} \phi}= & (1+\theta)\left(|\cos (\phi)|^{\theta}+|\sin (\phi)|^{\theta}\right)^{-1 / \theta-2}|\cos (\phi)|^{\theta-1}|\sin (\phi)|^{\theta-1} \\
& \times\left(\eta 1_{\{\cos (\phi) \sin (\phi)>0\}}+(1-\eta) 1_{\{\cos (\phi) \sin (\phi)<0\}}\right),
\end{aligned}
$$

and (21) applies. Such densities are shown in Figure 3. 
Spectral density of the Clayton

PLM with respect to $|\cdot|_{2}, \eta=1$

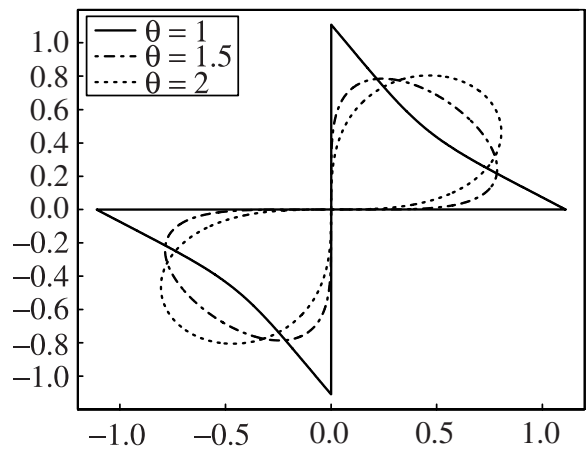

Spectral density of the Clayton

PLM with respect to $|\cdot|_{1}, \eta=1$

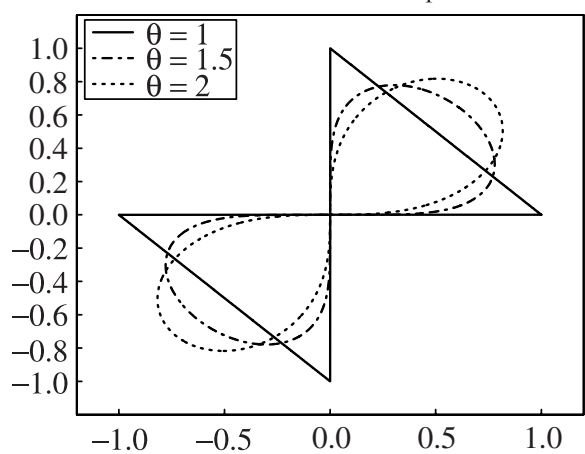

Spectral density of the Clayton

PLM with respect to $|\cdot|_{\infty}, \eta=1$

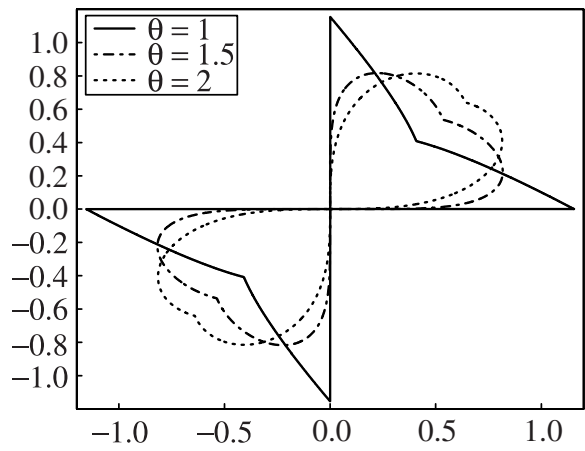

Spectral density of the Clayton

PLM with respect to $|\cdot|_{2}, \theta=2$

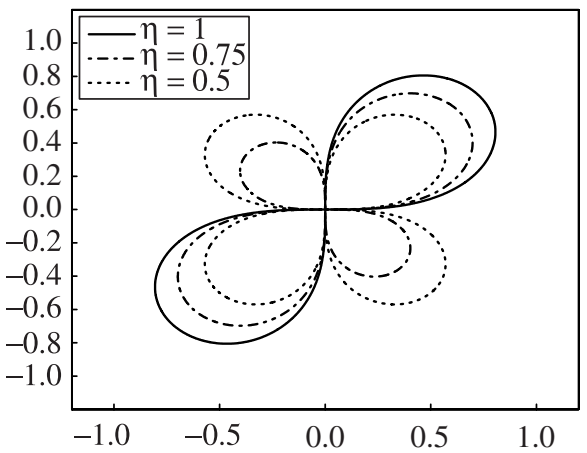

Spectral density of the Clayton

PLM with respect to $|\cdot|_{1}, \theta=2$

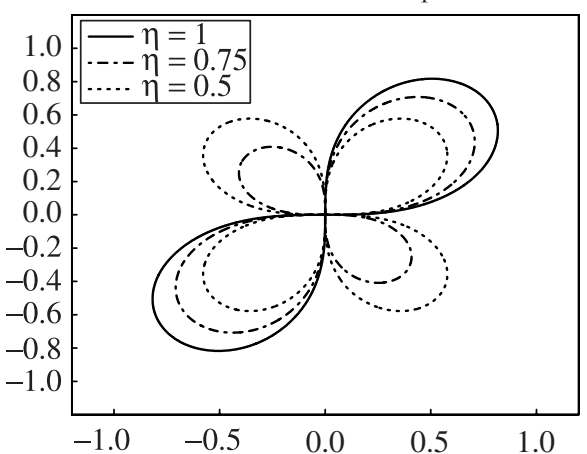

Spectral density of the Clayton

PLM with respect to $|\cdot|_{\infty}, \theta=2$

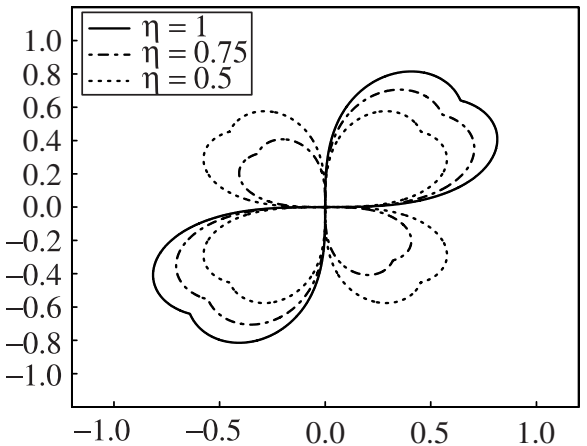

FIGURE 3: Basrak graphs of the spectral densities $\mu_{\mathbb{S}_{2}}(\mathrm{~d} \phi) / \mathrm{d} \phi$ (top row), $\mu_{\mathbb{S}_{1}}(\mathrm{~d} \phi) / \mathrm{d} \phi$ (middle row), and $\mu_{\mathbb{S}_{\infty}}(\mathrm{d} \phi) / \mathrm{d} \phi$ (bottom row) of the Clayton PLC for different parameter values of $\theta>0$ and $\eta \in[0,1]$ as given in $(18)$ on $[0,2 \pi)$.

We visualize the Clayton PLC $\bar{\Gamma}_{\eta, \theta}$ in Figure 2 for $\eta=1$ (i.e. joint jumps are always in the same direction) and different parameter values $\theta>0$. We see that, for increasing parameter $\theta$, the PLC values increase. This is reasoned by the increase of mass near $\pi / 4$. If $\theta$ decreases, the mass of $\tilde{\mu}_{\mathbb{S}}$ moves near to the axes and the PLC values decrease. 


\subsection{A nonhomogeneous Pareto Lévy measure}

Example 13. (Example 5 continued: nonhomogeneous PLM.) $\Gamma_{\eta, \zeta}$ has the density

$$
\begin{aligned}
\Gamma_{\eta, \zeta}\left(\mathrm{d} x_{1}, \mathrm{~d} x_{2}\right)= & \frac{2 \operatorname{sgn}\left(x_{1} x_{2}\right)+\zeta x_{1} \operatorname{sgn}\left(x_{2}\right)+\zeta x_{2} \operatorname{sgn}\left(x_{1}\right)+\zeta^{2} x_{1} x_{2}}{\left(\left|x_{1}\right|+\left|x_{2}\right|+\zeta\left|x_{1} x_{2}\right|\right)^{3}} \\
& \times\left(\eta 1_{\left\{x_{1} x_{2}>0\right\}}-(1-\eta) 1_{\left\{x_{1} x_{2}<0\right\}}\right) \mathrm{d} x_{1} \mathrm{~d} x_{2} .
\end{aligned}
$$

Transforming $\Gamma_{\eta, \zeta}$ to polar coordinates yields (20), where $\tilde{\mu}_{\mathbb{S}}=\tilde{\mu}_{\mathbb{S}}^{r}(\mathrm{~d} \phi)$ is given by

$$
\begin{aligned}
\frac{\tilde{\mu}_{\mathbb{S}}^{r}(\mathrm{~d} \phi)}{\mathrm{d} \phi}= & \frac{2 \operatorname{sgn}(\cos \phi \sin \phi)+\zeta r \cos \phi \operatorname{sgn}(\sin \phi)+\zeta r \sin \phi \operatorname{sgn}(\cos \phi)+\zeta^{2} r^{2} \cos \phi \sin \phi}{(|\cos \phi|+|\sin \phi|+\zeta r|\cos \phi \sin \phi|)^{3}} \\
& \times\left(\eta 1_{\{\cos \phi \sin \phi>0\}}-(1-\eta) 1_{\{\cos \phi \sin \phi<0\}}\right) .
\end{aligned}
$$

$\tilde{\mu}_{\mathbb{S}}^{r}$ depends on the radius and decreases for increasing $r$; see Figure 4.

Figure 5 shows the logarithmic PLM densities $\Gamma_{\eta, \theta} \circ T(r, \mathrm{~d} \phi)$ for $(\eta=1$ and $\theta=1)$ and $\Gamma_{\eta, \zeta} \circ T(r, \mathrm{~d} \phi)$ for $(\eta=1$ and $\zeta=0.001)$ for three different values of the radius $r$. We see

Logarithmized density $\Gamma_{\eta, \theta}^{\phi}(\mathrm{d} \phi) / \mathrm{d} \phi$ for $\eta=1$ and $\theta=1$

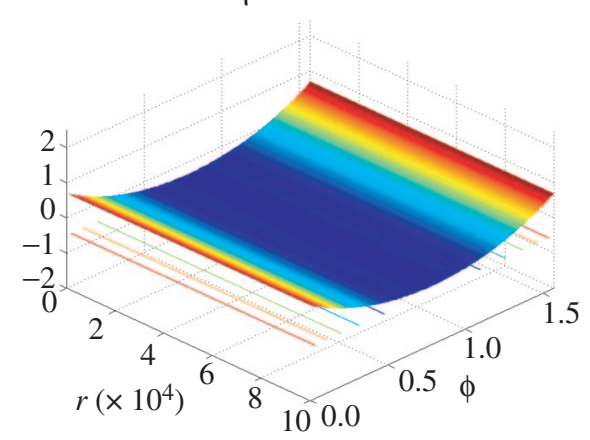

Logarithmized density $\Gamma_{\eta, \zeta}^{r, \phi}(\mathrm{d} \phi) / \mathrm{d} \phi$ for $\eta=1$ and $\zeta=0.001$

FIGURE 4: Densities $\tilde{\mu}_{\mathbb{S}}(\mathrm{d} \phi) / \mathrm{d} \phi$ (left) given in (22) (constant for all $r$ ) and $\tilde{\mu}_{\mathbb{S}}^{r}(\mathrm{~d} \phi) / \mathrm{d} \phi(r i g h t)$ given in

FIGURE 4: Densities $\mu_{\mathbb{S}}(\mathrm{d} \phi) / \mathrm{d} \phi($ left $)$ given in (22) (constant for 2 (23) (decreases with $r$ ).
(2)
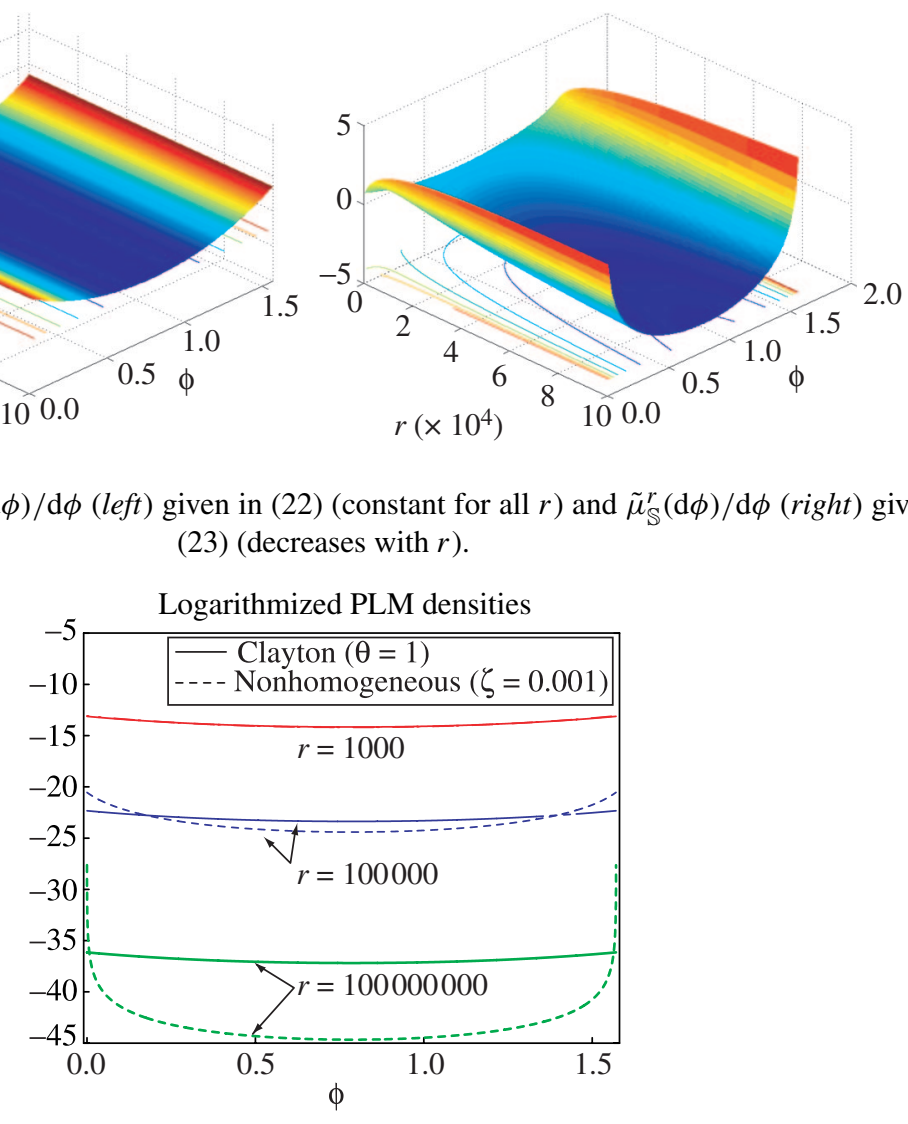

Figure 5: Logarithmic PLM densities of the Clayton PLM $\Gamma_{\eta, \theta}$ for $\eta=1$ and $\theta=1$ (shape independent of $r$ ), and the nonhomogeneous PLM $\Gamma_{\eta, \zeta}$ for $\eta=1$ and $\zeta=0.001$ (shape changes with $r$ ) for three different values of the radius $r$. 
that, for small $r$, both densities are almost identical. When $r$ increases, the Clayton density decreases uniformly for all angles $\phi$, but the nonhomogeneous density decreases strongly for angles near $\pi / 4$, and weakly for angles near 0 and $\pi / 2$.

Figure 6 shows the PLC $\bar{\Gamma}_{\|}$and $\bar{\Gamma}_{\eta, \zeta}$ for different parameter values of $\eta \in[0,1]$ and $\zeta>0$. We see that, for small values of $\zeta$, the PLM $\Gamma_{\eta, \zeta}$ is similar to the Clayton PLM $\Gamma_{\eta, \theta}$ with $\theta=1$. For increasing parameter $\zeta$, the PLC values become smaller and converge to independence.
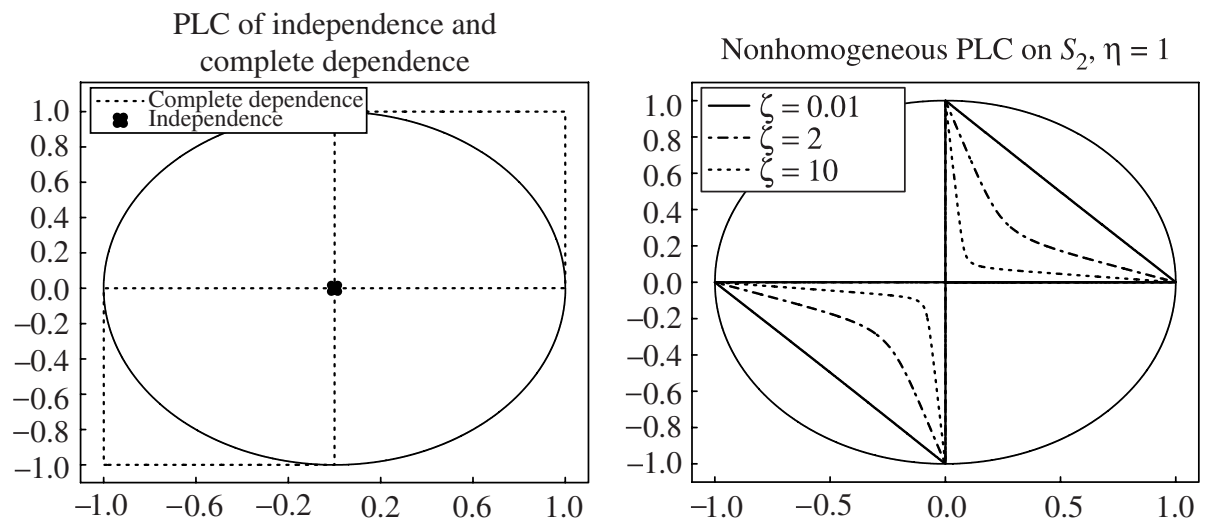

Nonhomogeneous PLC on $S_{2}, \zeta=0.001$
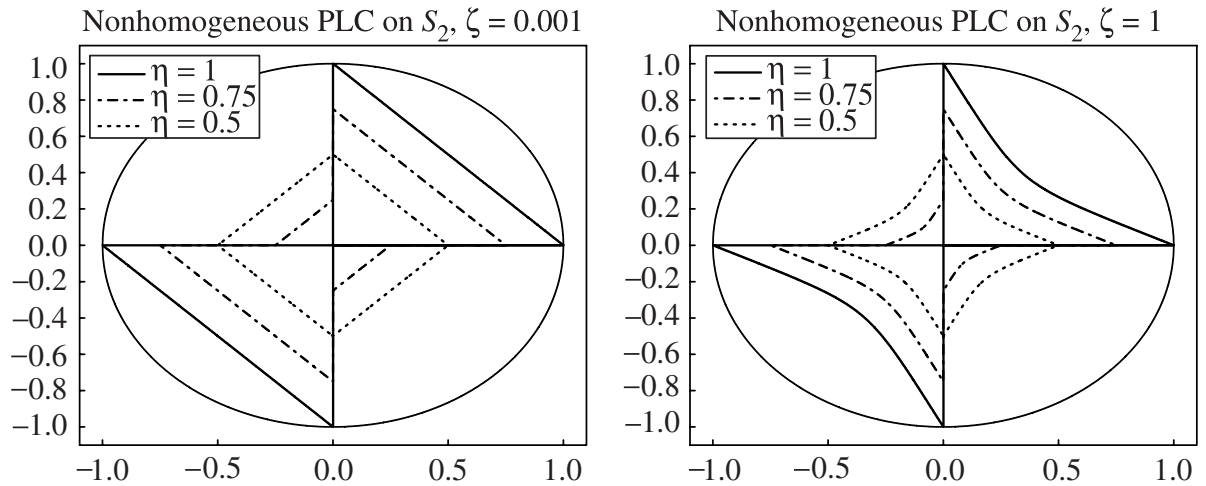

Nonhomogeneous PLC on $S_{2}, \zeta=2$
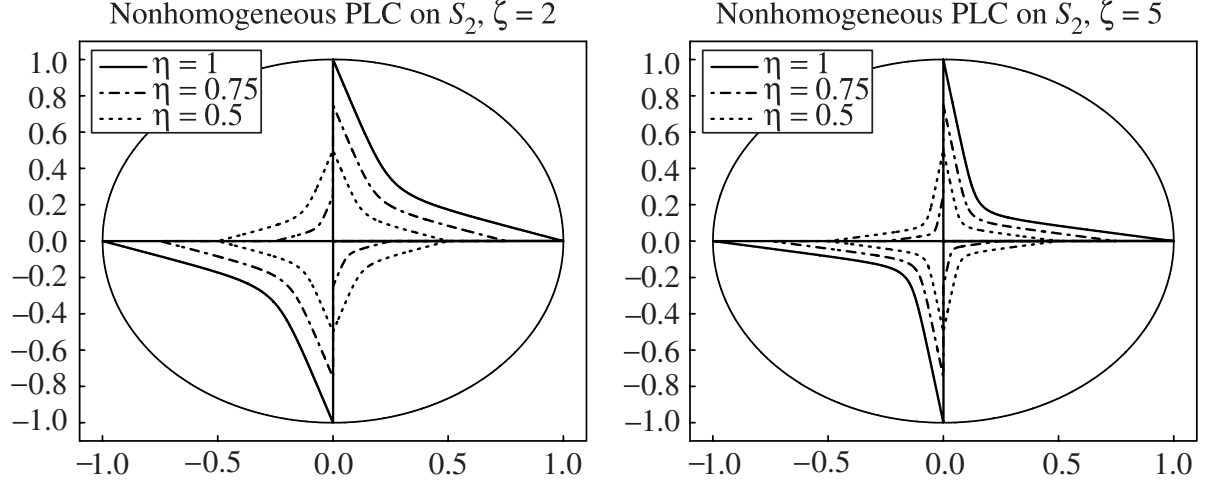

FiguRE 6: The diagrams show the PLC $\bar{\Gamma}_{\perp}, \bar{\Gamma}_{\|}$, and $\Gamma_{\eta, \zeta}$ for different parameter values $\eta \in[0,1]$ and $\zeta \in(0, \infty)$. 


\section{Acknowledgements}

I. E. gratefully acknowledges support by the Deutsche Forschungsgemeinschaft through the graduate programme Angewandte Algorithmische Mathematik at the Technische Universität München. C. K. thanks the Institute for Advanced Study of the Technische Universität München (TUM-IAS) for financial support.

\section{References}

[1] Barndorff-Nielsen, O. E. And Lindner, A. M. (2007). Lévy copulas: dynamics and transforms of Upsilon type. Scand. J. Statist. 34, 298-316.

[2] BasraK, B. (2000). The sample autocorrelation function of non-linear time series. Doctoral Thesis, Rijksuniversiteit Groningen.

[3] Basrak, B., Davis, R. A. And Mikosch, T. (2002). A characterization of multivariate regular variation. Ann. Appl. Prob. 12, 908-920.

[4] Böcker, K. AND KlÜPPelberg, C. (2010). Multivariate models for operational risk. Quant. Finance 10, 855869.

[5] Bregman, Y. and Klüppelberg, C. (2005). Ruin estimation in multivariate models with Clayton dependence structure. Scand. Actuarial J. 2005, 462-480.

[6] Cont, R. and Tankov, P. (2004). Financial Modelling with Jump Processes. Chapman \& Hall/CRC, Boca Raton, FL.

[7] De Haan, L. And Lin, T. (2001). On convergence toward an extreme value distribution in C[0, 1]. Ann. Prob. 29, 467-483.

[8] EdER, I. (2009). First passage events and multivariate regular variation for dependent Lévy processes with applications in insurance. Doctoral Thesis, Technische Universität München.

[9] Eder, I. AND KlüPPElberg, C. (2009). The first passage event for sums of dependent Lévy processes with applications to insurance risk. Ann. Appl. Prob. 19, 2047-2079.

[10] Esmaeili, H. AND KlÜPPelberg, C. (2010). Parameter estimation of a bivariate compound Poisson process. Insurance Math. Econom. 47, 224-233.

[11] Esmaeili, H. AND KlüPPelberg, C. (2011). Parametric estimation of a bivariate stable Lévy process J. Multivariate Anal. 102, 918-930.

[12] Esmaeili, H. ANd KLÜPPelberg, C. (2011). Two-step estimation of a multivariate Lévy process. Submitted.

[13] Giné, E., Hahn, M. G. and Vatan, P. (1990). Max-infinitely divisible and max-stable sample continuous processes. Prob. Theory Relat. Fields 87, 139-165.

[14] Hult, H. And LindsKog, F. (2005). Extremal behavior of regularly varying stochastic processes. Stoch. Process. Appl. 115, 249-274.

[15] Hult, H. ANd Lindskog, F. (2006). On regular variation for infinitely divisible random vectors and additive processes. Adv. Appl. Prob. 38, 134-148.

[16] Hult, H. and Lindskog, F. (2006). Regular variation for measures on metric spaces. Publ. Inst. Math. 80, 121-140.

[17] Hult, H. AND LindSKog, F. (2007). Extremal behavior of stochastic integrals driven by regularly varying Lévy processes. Ann. Prob. 35, 309-339.

[18] Joe, H. (1997). Multivariate Models and Dependence Concepts. Chapman \& Hall/CRC, London.

[19] Kallenberg, O. (1983). Random Measures, 3rd edn. Akademie, Berlin.

[20] Kallsen, J. AND TANKov, P. (2006). Characterization of dependence of multidimensional Lévy processes using Lévy copulas. J. Multivariate Anal. 97, 1551-1572.

[21] KlüpPelberg, C. And Resnick, S. I. (2008). The Pareto copula, aggregation of risks, and the emperor's socks. J. Appl. Prob. 45, 67-84.

[22] Nelsen, R. (2006). An Introduction to Copulas, 2nd edn. Springer, New York.

[23] Resnick, S. I. (1987). Extreme Values, Regular Variation, and Point Processes. Springer, New York.

[24] Resnick, S. I. (2007). Heavy-Tail Phenomena. Springer, New York.

[25] Samorodnitsky, G. And TaquU, M. S. (1994). Stable Non-Gaussian Random Processes. Chapman \& Hall, New York.

[26] Sato, K.-I. (1999). Lévy Processes and Infinitely Divisible Distributions. Cambridge University Press.

[27] UeltzhöFer, F. A. J. AND KlüPpelberg, C. (2011). An oracle inequality for penalised projection estimation of Lévy densities from high-frequency observations. J. Nonparametric Statist. 23, 967-989. 